\title{
Dynamic Moment Model for Numerical Simulation of a 6 DOF Plate Trajectory around an Aircraft
}

Kevin Ignatowicz (corresponding author), École de technologie supérieure, 1100 NotreDame Street West, Montreal H3C1K3, Quebec, Canada.

kevin.ignatowicz.1@ens.etsmtl.ca

François Morency, Ph.D., École de technologie supérieure, 1100 Notre-Dame Street

West, Montreal H3C1K3, Quebec, Canada. francois.morency@etsmtl.ca

Pierre Lopez, École de technologie supérieure, 1100 Notre-Dame Street West, Montreal

H3C 1K3, Quebec, Canada. plopez003@enseirb-matmeca.fr

\begin{abstract}
Ice shedding represents a threat to aircraft safety since ice blocks can strike rear components or can be ingested by engines. The accuracy of current numerical methods for predicting ice block paths in the design phase of an aircraft still need improvement. For the verification and validation of new trajectory calculation methods, shed blocks can be modelled for simplification as sphere or 6 Degree-Of-Freedom (6 DOF) plates. The objective of this paper is to propose a mathematical model for the dynamic moments of the plates and to use it to numerically simulate ice block paths. The results will be useful for verifying high-fidelity methods. Equations of motion in a Lagrangian frame are presented together with the correlations to be used for the aerodynamic coefficients of the ice blocks. The plate model involves the quaternions and a dynamic moment coefficient function of the angular velocity. After the model is validated with test cases obtained from the literature, the trajectories around the blended wing body will be plotted. The sensitivity of the trajectories and footprints to the chosen dynamic moment model will be highlighted.
\end{abstract}




\section{Introduction}

Weather-related aircraft accidents due to icing between 1967 and 2004 were at the root of about $50 \%$ of recommendations published by safety authorities covering icing hazards (Skeen Jr and Reed 2004). Ice accretion on an aircraft can impact its aerodynamic performance and handling or obstruct its sensors. This can lead to catastrophes, such as the Rio-Paris Air France accident in 2009, which was caused by icing on the Pitot Tubes (Conversy et al 2014). According to the International Air Transport Association (I.A.T.A.) 2015 Safety Report (I.A.T.A. 2016), recent statistics show that ice-related issues account for $17 \%$ of all in-flight loss of control. De-icing systems, including pneumatic boots for example, are mounted on aircrafts to remove accreted ice. Nevertheless, removed ice particles are shed, and can in turn strike the airplane or can be ingested by aft-mounted engines. Consequently, it is crucial to take icing hazards and the trajectories of these ice blocks shed into account in the design phase of an airplane.

Shedding and trajectory simulations are challenges when studying the de-icing phenomenon in aeronautics and in wind engineering.

In wind engineering, the simulation of flying debris trajectories in a flow field has been studied extensively. 2D models have been developed, for example, by Holmes (2004), but recent efforts have been concentrated on simulating a 3D phenomenon for more realistic predictions (Kordi and Kopp 2009). For the plate ice piece, Richards et al (2008) present a methodology coupling experimental data with numerical simulations. Baker (2007) classifies wind-borne debris under two main categories: compact debris and sheet debris. The aerodynamic forces and moments on sheet debris change with the orientation and 
rotation speed. The forces and moments can be modelled with static and dynamic coefficients.

In aeronautics, ice fragments result from ice accumulation on unprotected surfaces or ice growth on aircraft surfaces due to delayed activation of ice protection systems. These fragments can take various shapes, but are all categorized as sheet debris. Accurate ice trajectory simulation methods are yet to be developed. Currently, two trajectory simulation methods are available for use, low fidelity methods and high-fidelity methods, with the latter being the more recent.

Low fidelity methods assume that ice fragments do not affect the flow field. Papadakis et al (2007) simulate ice shedding by coupling numerical computerized simulations with experimental wind tunnel data. The aerodynamic coefficients of ice fragments are determined using experimental data, after which they are imported into the numerical model. High-fidelity methods take into account ice interaction with the flow, and require the use of emerging simulation tools to tightly combine flight mechanics and aerodynamics.

To validate the new high-fidelity methods (Beaugendre et al 2018), databases and models are available for plates and spheres. Because ice fragments take various random shapes, with geometric databases often confidential, specific numerical or experimental databases are needed, but no such databases are available in the literature (Beaugendre et al 2018). The plate trajectory calculation assumes a major shape simplification of the ice fragment, but it allows sharing results between different research groups. Such 6 DOF trajectory calculations are akin to what is carried out for missile guidance. The common aspect between missiles and ice blocks is their motion only based on aerodynamic, inertia and 
gravitational forces. The aerodynamic coefficients for missiles are interpolated in a database to be evaluated at specific pitching, rolling and yawing angles (Sefastsson 2016). Databases are necessary for complex shapes that don't have analytical aerodynamic correlations, such as missiles (Nichols 1977) or random ice blocks. Nevertheless, databases can be replaced by analytical solutions (Paramasivam et al 2015) for simple shapes like the plate or spherical debris studied in the present paper. It is then easier to compare results on basic shapes with other researches groups, since an analytical solution doesn't rely on databases that can vary from a group to another. In addition, databases are often confidential and not published, making it difficult to compare the results.

While the static aerodynamic coefficients of a plate are well established (Hoerner 1965), no consensus has emerged on the dynamic coefficients. Moreover, the latter are also difficult to measure in wind tunnel experiments. The dynamic moment is one of the most sensitive parameters for the rotating plate, and several moment's models can be considered. Thus, several approaches have emerged in the literature:

- a constant dynamic moment (Papadakis et al 2007);

- a piecewise linear dynamic moment with respect to the angular velocity (Tachikawa 1983);

- a hybrid model in which the dynamic moment depends on the relative flow velocity, and is pseudo-quadratic with respect to the angular velocity, i.e., each angular velocity component is multiplied by the magnitude of the angular velocity (see Eq. (6)) (Richards et al 2008).

A study of the sensitivity of the numerical 3D trajectories of sphere and plate ice pieces to the dynamic model will help in validating high-fidelity methods. These methods are based 
on different models, and should generally give results falling within a given uncertainty range. In the present study, attention is paid to the footprints of the trajectories in a transverse plane, near the rear of an airplane. The innovation, and main objective of this paper, will be to propose a model for the dynamic moment acting on the rotating plate and implement it in a Lagrangian trajectory code. The dynamic moment model proposed herein is a $3 \mathrm{D}$ extension of a $2 \mathrm{D}$ model proposed in the $80 \mathrm{~s}$ (Tachikawa 1983). This model is based on a limiting value for the angular velocity and a tri-linear dependence of the moment magnitude regarding the angular velocity, since it presents three linear intervals. One simplification of the present model is to neglect the influence of small ice particles on the flow field. The flow field around a clean aircraft without ice particles is kept during the entire process. For better fidelity and future improvements of the model, fluid-structure interaction may be needed to model the changes in the flow field induced by the moving particles (He et al 2018). Nevertheless, by neglecting the solid effects on the flow, we could expect a computational time reduced by a factor 150 as compared to a fully coupled fluidstructure interaction simulation (Olivier and Dumas 2012), and shows less than an $8 \%$ difference with experimental results, as illustrated in the results section. By comparing the footprints obtained with the dynamic moment proposed by Richards et al (2008) and the new dynamic moment model, the impact of the dynamic moment model on the plate trajectory path and their footprints in a given cross-plane can be estimated.

The equations of motion of ice pieces use aerodynamic correlations to determine the drag coefficient for the sphere, as well as the normal force coefficient for the plate. The numerical method solves the equations of motion with a third-order adaptive Runge-Kutta method. 
The methodology section focuses on the model and the modules needed to perform a trajectory simulation. The theory section gives more details about the equations and the mathematical concepts involved in the simulations. After a validation against the work of Richards et al (2008), for the plate, and of Holmes (2004), for the sphere, a discussion is carried out to compare the dynamic moment proposed by Richards et al (2008) to the 3D tri-linear model based on the work of Tachikawa (1983). The code will then be used to perform 3D simulations around a complete blended-wing-body (BWB) aircraft, and 3D plots and footprint distributions using both dynamic moment approaches will highlight the differences.

\section{Methodology}

This section presents the steps required to perform a trajectory simulation in a flow field. After an examination of the configuration layout used for the BWB studied in the present paper, the algorithm of the trajectory code will be detailed.

\section{Geometry and set-up}

This sub-section describes the geometry and set-up used to conduct an ice particle trajectory simulation in a given flow field. The flow field is the one around the BWB obtained by Computational Fluid Dynamics (CFD) (Velázquez Salazar et al 2017). The BWB presents a length of $25 \mathrm{~m}$ and a half-span of $14 \mathrm{~m}$. Its visual aspect is shown in Fig.1. 


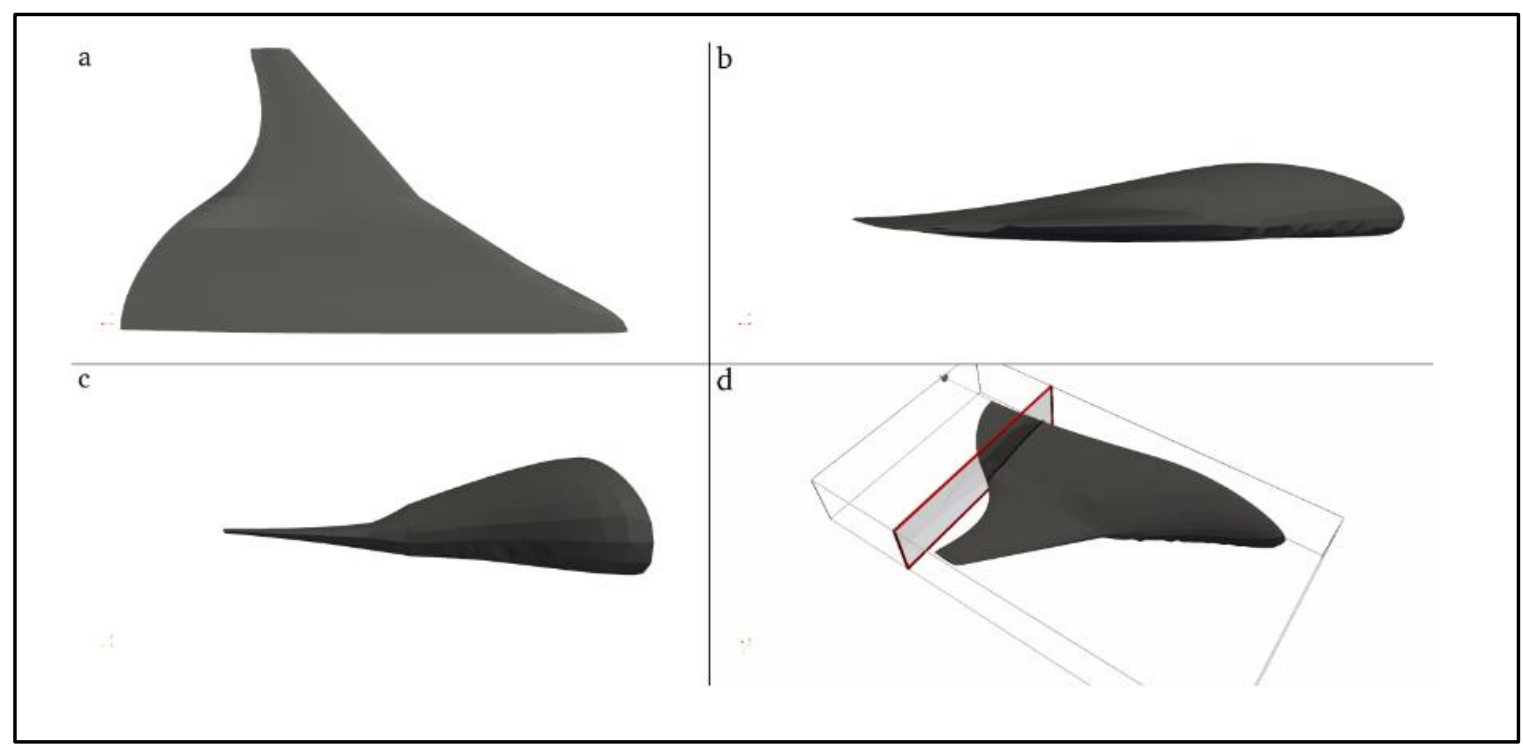

Fig.1 Different views of the BWB geometry: a) top view, b) side view, c) rear view, d) location of the cross-flow plane where the footprints of trajectories will be investigated

The CFD simulations were performed by Velázquez Salazar et al using the RANS solver of the SU2 software (Palacios et al 2015). The turbulence model used was the SpalartAllmaras model. The airplane configuration was a final approach at low speed, at 8 degrees of angle of attack. The following freestream variables were used:

- Mach number: 0.19;

- Temperature: $288 \mathrm{~K}$;

- Reynolds number: 73.7e6, based on freestream velocity and a reference length of $15.9 \mathrm{~m}$, corresponding to the mean chord length of the BWB.

The domain used for the trajectory simulations was a 40 by 20 by $40 \mathrm{~m}$ box clipped inside the original semi-spherical CFD domain of 1 million cells. The discretized clipped computation domain was an unstructured mesh composed of 357,129 cells. It should be noted that a finer mesh with more cells is needed, especially near walls, for an effective 
aerodynamic performance prediction. Nevertheless, this coarse base was enough for the verification of the trajectory code, as ice trajectories are mostly located outside the boundary layer and wake areas. Fig.2 presents the mesh and the computed streamlines around the BWB model. Ice particles are inserted at several locations near the wing leading edge.

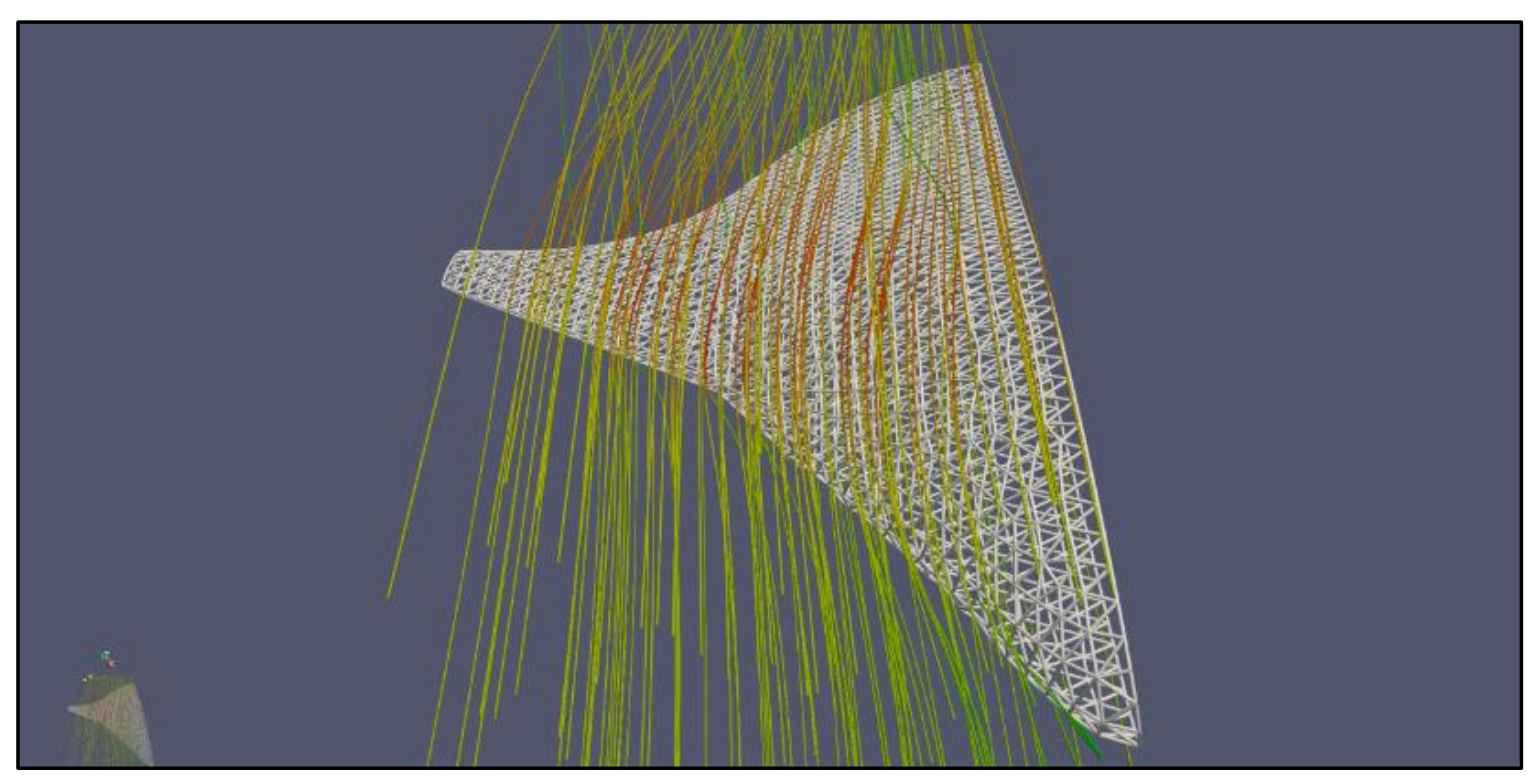

Fig.2 Surface mesh of the CFD model and streamlines around the geometry

The CFD data file was used as an input for the trajectory code. The BWB geometry was chosen to demonstrate the ability of the code to perform trajectory simulation. In practice, the trajectory code can perform simulations in every CFD flow field or uniform flow field. The following assumptions were made about the physics of the system:

- The CFD simulation is performed around a half-aircraft;

- The flow field is calculated around a clean aircraft, without any ice accretion;

- Ice particles do not influence the flow field (one-way coupling);

- Ice particles are represented either as spheres or as plates; 
- The rotation of the spherical ice particle is not taken into account;

- Only the normal force is taken into account for the plate.

\section{Trajectory code}

A 2D trajectory code (Védie et al 2016) is used as the starting point for the present work, which extends its capabilities to 3D simulations with 6 DOF. The trajectory code is divided into modules. A first module brings together all the physical constants, variables and initial conditions. The other modules are part of the main program. One module contains all the governing equations of motion, plus the quaternions and the moment equations, in the case of the plate. These equations are solved by a module using MATLAB Ode23 (Shampine and Reichelt 1997). At each time step, an interpolation module computes the local velocity components by linear interpolation in the CFD flow field (see next section). Finally, the last module performs the outputs and graphs. The outputs returned are 3D plots of ice block trajectories around the $\mathrm{BWB}$, and a $2 \mathrm{D}$ footprint of trajectories in an $X$-normal plane located $20 \mathrm{~m}$ downstream from the front end of the geometry. The algorithm of the code is similar to the one used by Papadakis et al (2007). Fig.3 presents the structure of the trajectory code. 


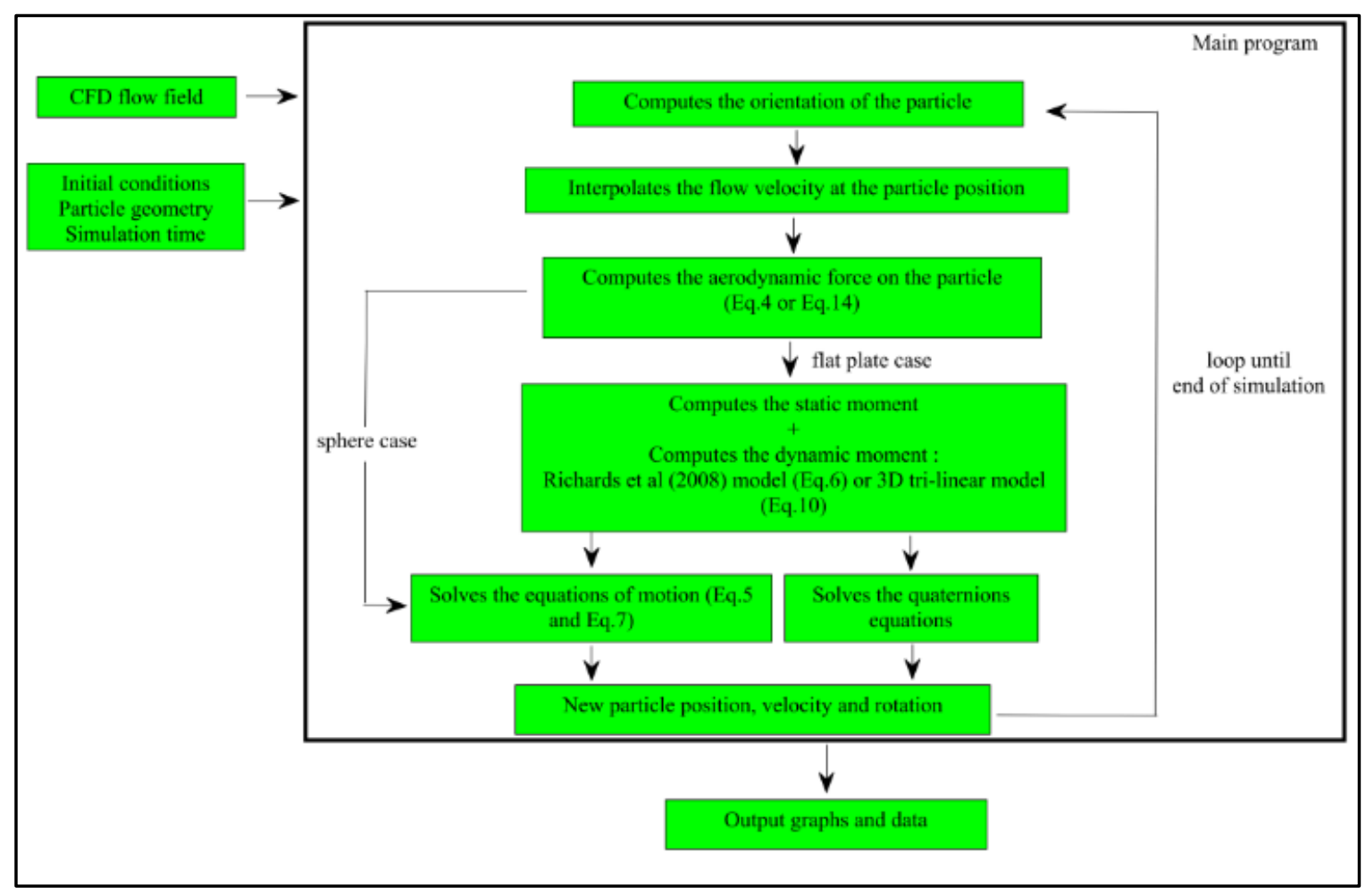

Fig.3 Algorithm of the trajectory code

\section{Theory and calculation}

In this section, the mathematical models used in each module described in the methodology section are presented. Trajectory calculations are centred on the Newton's laws of motion. The challenges in the present case lay in modelling the aerodynamic forces and moments on the ice particle. Two different aerodynamic models were used, one for the plate and one for the sphere.

\section{Interpolation module}

This module is common for the plate and the sphere codes. Its aim is to calculate the velocity components in all three directions at every location $\left(X_{q}, Y_{q}, Z_{q}\right)$ in the domain, 
using a linear interpolation. To perform the interpolation, the scatteredInterpolant MATLAB function is used (Amidror 2002). No errors due to the interpolation method were seen during the sensitivity tests carried out. With the velocity computed at position $\left(X_{q}, Y_{q}\right.$, $Z_{q}$ ), the aerodynamic forces and moment can be computed using empirical correlations. A description of the interpolation module for 2D applications was presented in a previous paper (Ignatowicz and Morency 2017).

Plate

The plate is in a translational movement in all three directions, $X, Y$ and $Z$, in the global reference axis and in rotation about its three local rotating axes, $X_{p}, Y_{p}$ and $Z_{p}$. Its orientation at a given time is described by the three Euler angles, $\Psi, \theta$ and $\Phi$, corresponding to the $Z_{p}$, $Y_{p}$ and $X_{p}$ rotations (see Fig.4). The plate has a dimension $L$ in the $X_{p}$ direction, $l$ in the $Y_{p}$ direction, and a thickness $e$. The surface ratio is $S R=L / l$.

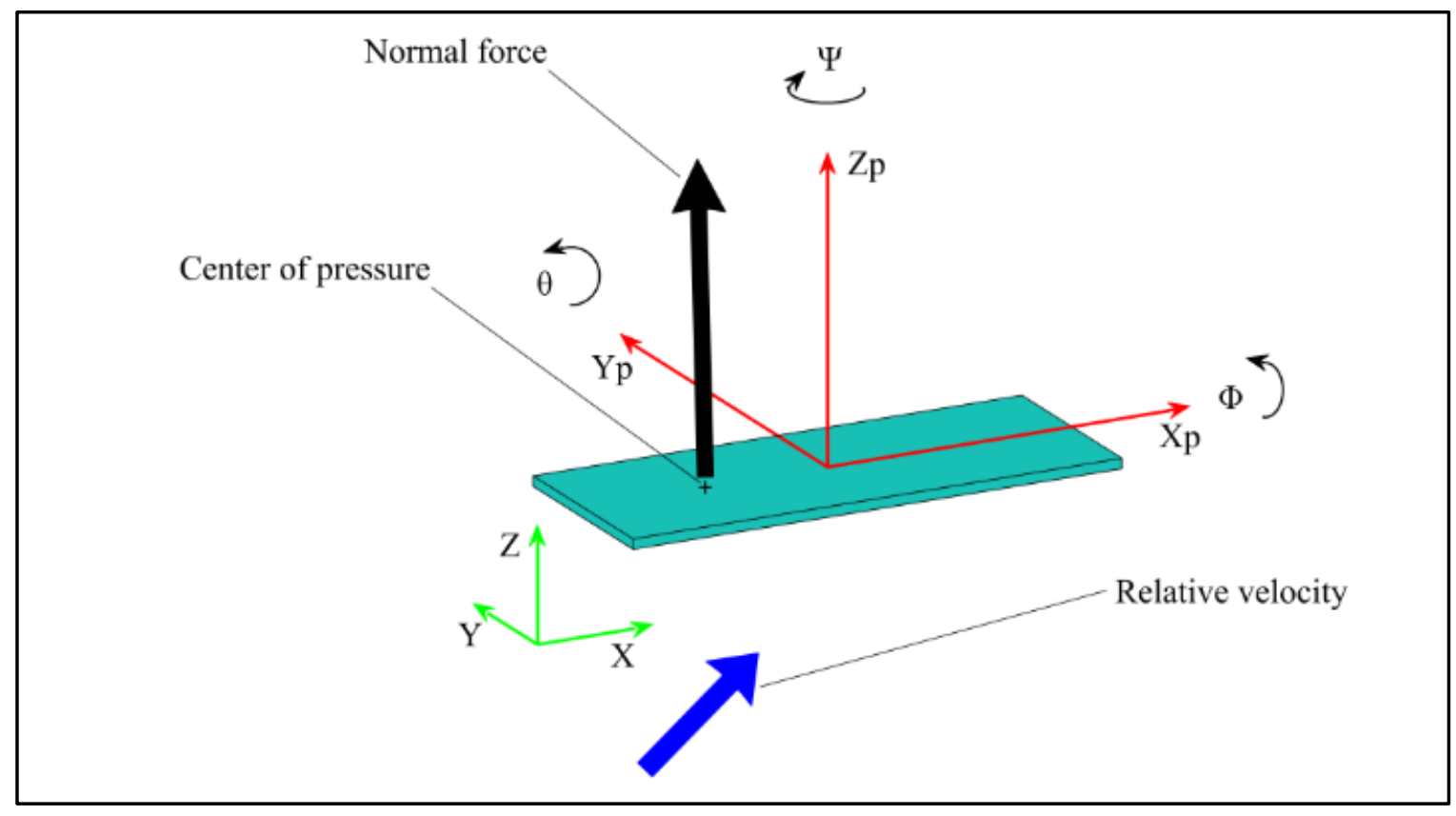

Fig.4 Representation of the plate motion 
To describe the plate orientation, Min Fu et al (2013) propose using the quaternions instead of the raw Euler angles in order to avoid some situations with singular values, called Gimbal locks. The quaternions are four scalars, $q_{1}, q_{2}, q_{3}$ and $q_{4}$, determined from the Euler angles.

These quaternions will also determine the matrix $\mathbf{R}_{\mathbf{q}}(\mathbf{q})$ that can change a vector from the global to the local reference axis formulation. The expression of $\mathbf{R}_{\mathbf{q}}(\mathbf{q})$ is detailed by Min Fu et al (2013).

The motion and rotation of the plate are mainly a function of the aerodynamic forces acting on it. At each query point $P_{q}$, the flow field has a velocity $\mathbf{V}=\left[U_{q} ; V_{q} ; W_{q}\right]$ in the global reference axis and the plate itself has a velocity $\mathbf{u}=[u ; v ; w]$ in the same global reference axis. To compute the aerodynamic forces acting on the particle, the relative velocity $\mathbf{V}_{\text {rel }}=$ $\left[u_{r} ; v_{r} ; w_{r}\right]$ is needed (1).

$$
\mathbf{V}_{\text {rel }}=\mathbf{V}-\mathbf{u}
$$

This relative velocity has to be changed to the local rotating axis formulation using the $\mathbf{R}_{\mathbf{q}}(\mathbf{q})$ matrix:

$$
\mathbf{V}_{\text {relm }}=\mathbf{R}_{\mathbf{q}}(\mathbf{q}) \mathbf{V}_{\text {rel }}=\left[u_{r m} ; v_{r m} ; w_{r m}\right]
$$

To be able to calculate the aerodynamic forces from this relative velocity, the two angles describing the orientation of the relative velocity in the mobile axis are computed: the angle of attack $\alpha$ and the side slip angle $\beta$ (see Fig.5 and Eq. (3) for their definitions). 


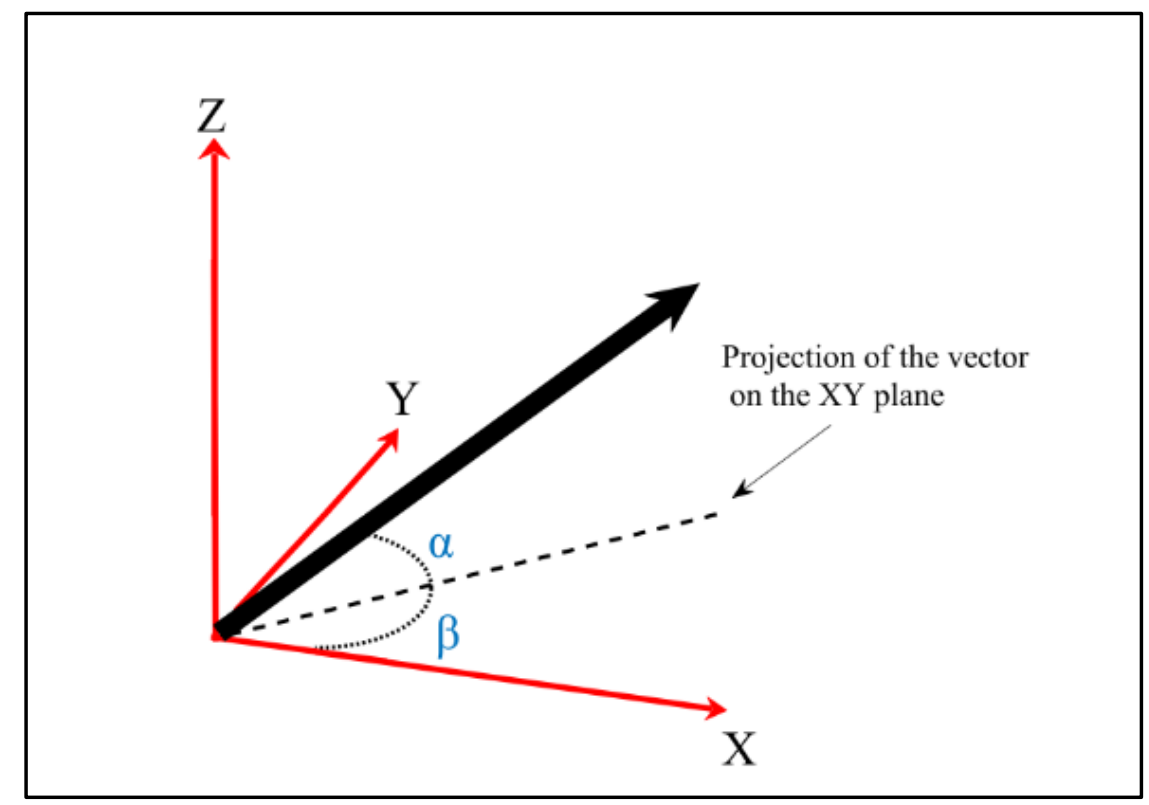

Fig.5 Angle of attack $\alpha$ and side slip angle $\beta$

$$
\begin{gathered}
\alpha=\operatorname{asin}\left(\frac{w_{r m}}{\left|\mathbf{V}_{\text {relm }}\right|}\right) \\
\beta=\operatorname{asin}\left(\frac{v_{r m}}{\left|\mathbf{V}_{\text {relm }}\right| \cos (\alpha)}\right)
\end{gathered}
$$

With these angles and the assumption of Richards et al (2008) that the only aerodynamic force is the normal force acting on the center of pressure, in the mobile axis system, the components of the force $\mathbf{F}_{\mathbf{P}}$ are:

$$
\begin{gathered}
F_{X P}=0 \\
F_{Y P}=0 \\
F_{Z P}=0.5 \rho\left|\mathbf{V}_{\text {relm }}\right|^{2} L l C_{N}
\end{gathered}
$$

where:

- $\quad \rho$ is the density of the air $\left(\mathrm{kg} / \mathrm{m}^{3}\right)$;

- $C_{N}$ is the normal coefficient. 
According to Richards et al (2008), for a plate, the normal force coefficient is determined as a function of $\alpha$ and $\beta$ by experimental tests in wind tunnel. These results are used to build a database. The normal coefficient is known for plates with a surface ratio $S R$ of 1,2 or 4. Fig.6 illustrates the $C_{N}$ for $S R=1$. The coordinates of the center of pressure are also given in the paper by Richards et al (2008).

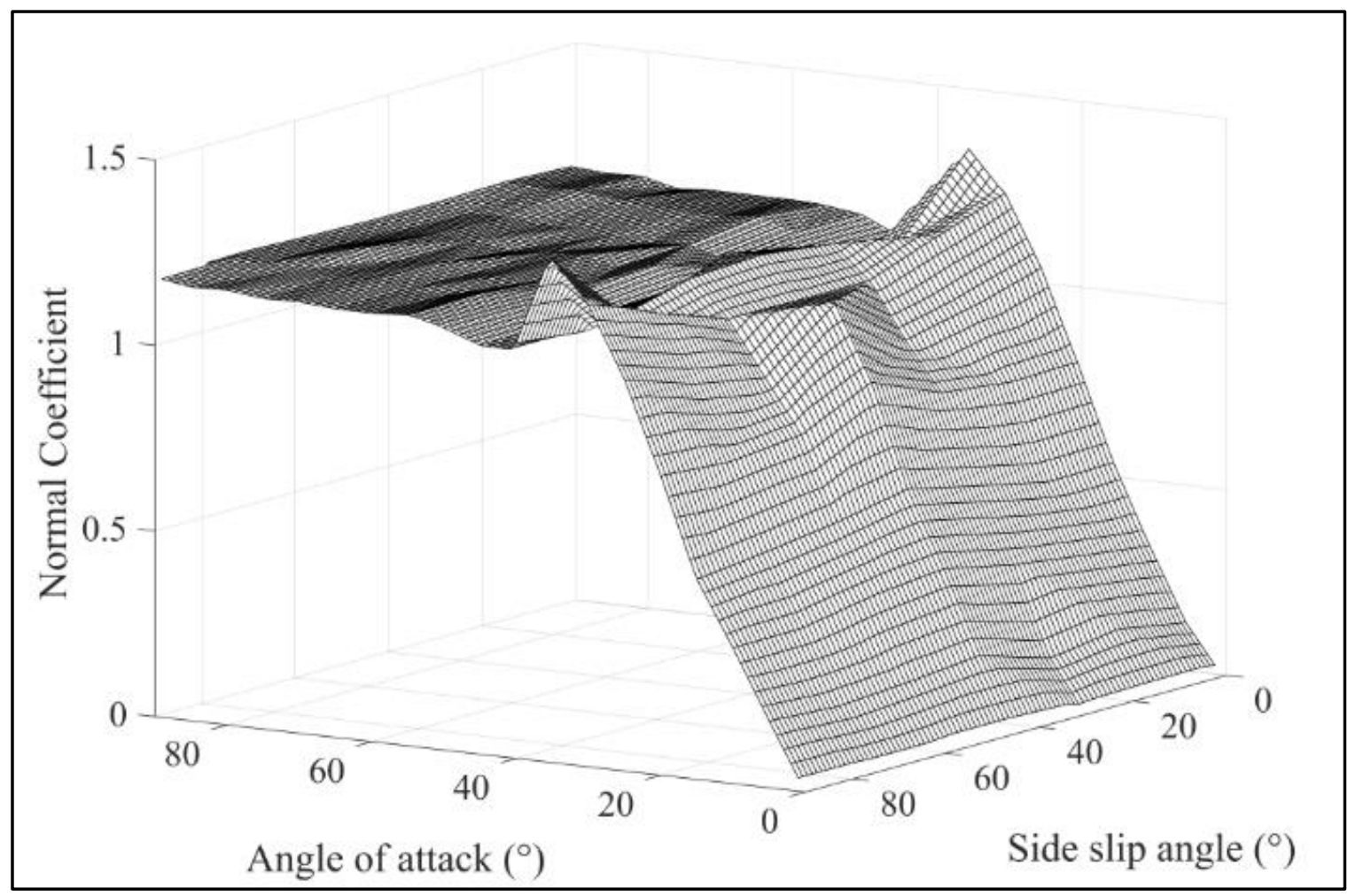

Fig.6 $C_{N}$ for a plate with $S R=1$, adapted from Richards et al (2008)

To determine the translational motion of the plate in the reference axis, the force $\mathbf{F} \mathbf{P}(4)$ is changed to the global reference axis formulation to solve the Newton equation of motion (5):

$$
m \dot{\mathbf{u}}=\mathbf{F}+m \mathbf{g}
$$


with: $\mathbf{F}=\mathbf{R}_{\mathbf{q}}^{-\mathbf{1}}(\mathbf{q}) \mathbf{F}_{\mathbf{p}}$ and $\mathbf{g}=[0 ; 0 ;-9.81]$

The dot stands for the temporal derivative, and $m$ is the mass of the ice particle calculated with a density of $917 \mathrm{~kg} / \mathrm{m}^{3}$.

The angular velocity vector, about $X_{p}, Y_{p}$ and $Z_{p}$, is $\mathbf{\Omega}=[P ; Q ; R]$. The static moment $\mathbf{M}$ of the plate at the center of gravity results from the force applied at the center of pressure.

To avoid an unlimited angular velocity for the plate, Richard et al (2008) propose a dynamic moment $\mathbf{M}_{\mathbf{d}}$ to be added to the previous static moment:

$$
\mathbf{M}_{\mathbf{d}}=0.5 \rho \mathbf{C}_{\mathrm{dm}}\left(\left|\mathbf{V}_{\text {relm }}\right|+|\boldsymbol{\Omega}| \frac{l}{2}\right) L l^{3} \boldsymbol{\Omega}
$$

with $\mathbf{C}_{\mathbf{d m}}=[-0.2 ;-0.185 ;-0.01]$ being the constant vector values selected to fit experimental results (Richards et al 2008).

The total moment $\mathbf{M}_{\mathbf{p}}$ is $\mathbf{M}+\mathbf{M}_{\mathbf{d}}$. Once the total moment and the matrix of inertia of the plate $\mathbf{I}$ are known, the conservation of angular momentum equation, with $\Lambda$ standing for the cross product of two vectors, is:

$$
\mathbf{I} \dot{\mathbf{\Omega}}=\mathbf{M}_{\mathbf{P}}-\boldsymbol{\Omega} \wedge \mathbf{I} \boldsymbol{\Omega}
$$

The dynamic moment of Eq. (6) proposed by Richards et al (2008) is constantly opposed to the angular velocity. As shown in the results section, this eventually stops the rotation of the plate in its flight and induces a translating motion. However, experimental video recordings by Tachikawa (1983) show that in most cases, a plate is either totally in translation or in rotation, or evolves from translation to rotation. The case where a rotating plate acquires a translation motion was not observed. 
The dynamic moment proposed by Tachikawa (1983) was a 2D model. The fundamental aspect of this model is that the plate has a maximum angular velocity, depending on the flow relative velocity and the geometry. This angular velocity $\omega_{0}$ is given by Eq. (8):

$$
\omega_{0}=\frac{2\left|\mathbf{V}_{\text {relm }}\right| K}{l}
$$

where:

- $\omega_{0}$ is the maximum angular velocity $(\mathrm{rad} / \mathrm{s})$;

- $K$ is the parameter measured experimentally: 0.32 for a square plate, 0.45 and 0.48 for an $e / l$ ratio of $5.3 \%$ and $2.9 \%$, respectively, for a rectangle plate (Tachikawa $1983)$

- $\quad l$ is the dimension of the plate perpendicular to the rotation axis (m).

The 2D dynamic moment proposed by Tachikawa (1983) has the generic expression given by Eq. (9), where $C_{m r}$ is the dynamic moment coefficient, and is scalar since there is only one rotation (see Fig.7 for its definition).

$$
\mathbf{M}_{\mathbf{d}}=\frac{1}{2} \rho\left|\mathbf{V}_{\text {relm }}\right|^{2} L l^{2} C_{m r}
$$

The dynamic moment model of Eq. (9) was used for 2D motions involving only one rotation perpendicular to the flow direction. The present work proposes an extension to 3D models involving three rotations. In practice, the rotation about the $Z_{p}$ axis presents a very small frontal area (i.e., le or $L e$ ). The dynamic moment coefficient can then be assumed to be 0 for that rotation. The 3D-adapted model proposes to define maximum angular velocity values for the two rotations about $X_{P}$ and $Y_{P}$ and the dynamic moment coefficients for both of them. The expression of the 3D dynamic moment is given by Eq. (10): 


$$
\mathbf{M}_{\mathbf{d}}=\frac{1}{2} \rho\left|\mathbf{V}_{\text {relm }}\right|^{2}\left[\begin{array}{c}
l^{2} L C_{m r x} \\
L^{2} l C_{m r y} \\
0
\end{array}\right]
$$

where $C_{m r x}, C_{m r y}$ and $C_{m r z}=0$, are the components of the 3D-extended dynamic moment coefficient $C_{m r}$. The 2D dynamic moment coefficient was experimentally determined by Tachikawa (1983) as a function of the ratio $\omega_{/} \omega_{0}$, where $\omega$ is the angular velocity of the plate (see Fig.7).

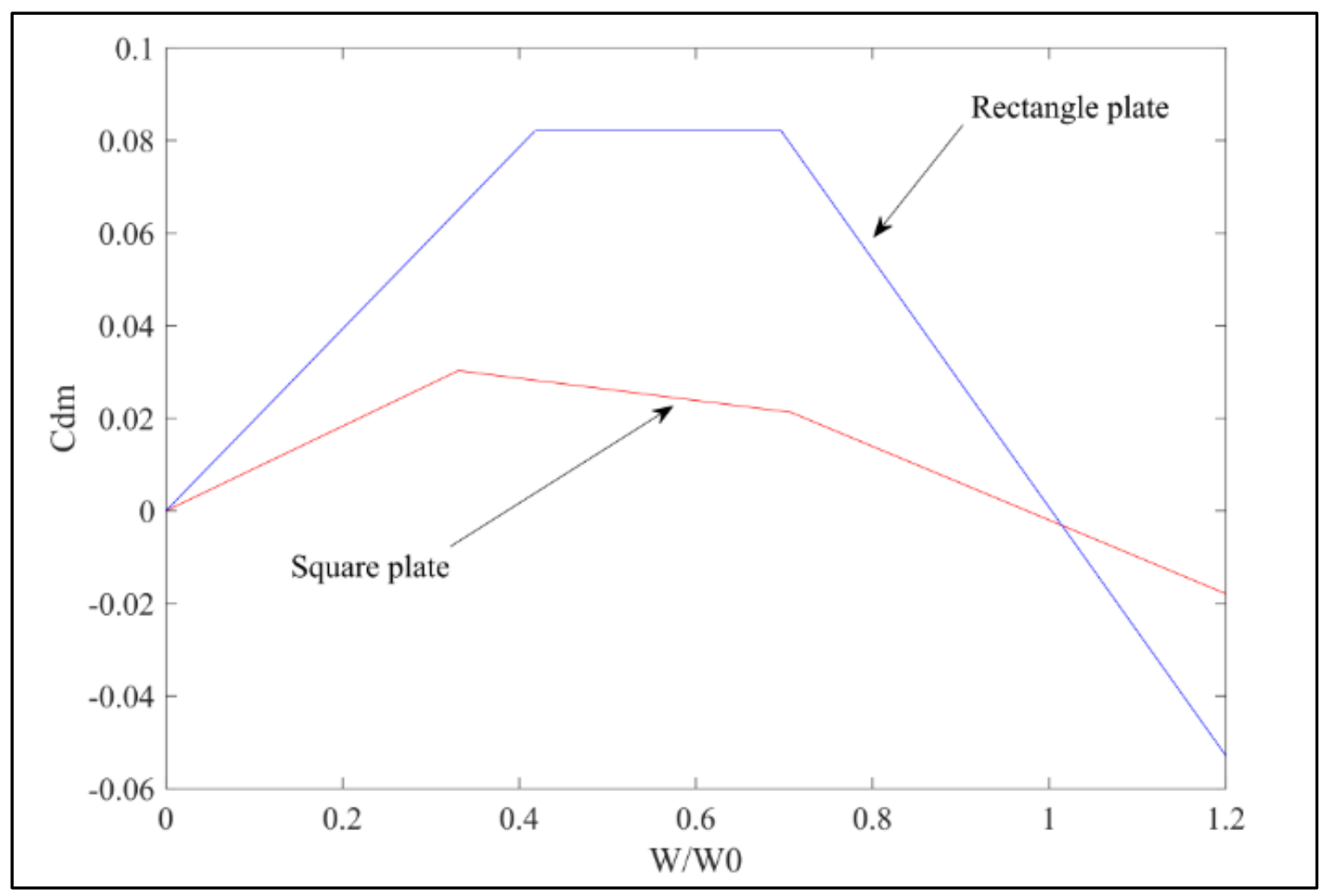

Fig.7 Dynamic moment coefficient as a function of $\omega / \omega_{0}$, adapted from Tachikawa (1983)

In the 3D adaptation, the $C_{m r}$ will be considered to be the same for the two rotations of the plate, meaning that the $\omega$ of Fig.7 stands for $P$ or $Q$. Following that, the maximum angular 
velocity values will be denoted as $P_{0}, Q_{0}$. For the square plate, the value of 0.32 for $K$ will be kept for the two rotations.

For the rectangle plate, the $K$ values determined by Tachikawa (1983) are 0.45 and 0.48 , depending on the e/l ratio, as shown earlier. Tachikawa determined these values for a rotation about the longest axis of the plate, $X_{p}$. In the present $3 \mathrm{D}$ extension, $K$ for the $X_{p}$ rotation is chosen to be a linear function of the ratio $e / l$, matching the values of Tachikawa for ratios of 2.9 and $5.3 \%$. This will allow the simulation of trajectories for plates with various thickness ratios. The $K$ value for the rotation about the $Y_{p}$ axis was taken as 0.4 as the plate can rotate faster about the $Y_{p}$ axis than about the $X_{p}$ axis due to a smaller length in the axis direction. This value of 0.4 is a hypothesis, and is an extrapolated value, based on the criterion that $K$ has to be greater in that case than in the square plate case. Finally, the maximum angular velocities are given by Eq. (11), where the expressions are extrapolated from Tachikawa (1983):

For a square plate: $P_{o}=Q_{o}=0.64 \frac{\left|\mathbf{V}_{\text {relm }}\right|}{L}$

$$
\begin{gathered}
\text { For a rectangle plate: } P_{o}=2\left(-1.25 \frac{e}{l}+0.516\right) \frac{\left|\mathbf{V}_{\text {relm }}\right|}{l} \\
\qquad Q_{o}=0.8 \frac{\left|\mathbf{V}_{\text {relm }}\right|}{L}
\end{gathered}
$$

And the dynamic moment coefficient is: 


$$
\mathbf{C}_{\mathbf{m r} 3 \mathrm{D}}=\left[\begin{array}{c}
\operatorname{sign}(P) F_{D M}\left(\frac{|P|}{P_{0}}\right) \\
\operatorname{sign}(Q) F_{D M}\left(\frac{|Q|}{Q_{0}}\right) \\
0
\end{array}\right]
$$

The linear by segments function of Fig. 7 is denoted $F_{D M}$. The absolute values and the sign function are used to allow positive and negative rotations, since the graphs of Fig.7 were experimentally obtained by Tachikawa for positive angular velocities. The assumption in the present work is that for a negative rotation, the value of the dynamic moment coefficient is the same, but of opposite sign. Eq. (8) shows that $K$ is one of the major parameters involved in the model. Nevertheless, a sensitivity analysis performed shows that varying $K$ has a minor impact on the trajectory. Setting $K$ at 0.30 instead of 0.32 (6\% variation ) for the square plate implies a variation of $1.3 \mathrm{~cm}$ in the trajectory path after $20 \mathrm{~m}$ travelled, representing an error of just above $0.06 \%$. For the same amount of variation of $K$, the conclusion is the same for the rectangle plate: differences in trajectories are less than $0.1 \%$.

By solving the translation, the moment and the quaternion equations simultaneously, the trajectory of the 6 DOF plate is computed in the flow field around the BWB. The six ordinary differential equations are solved with a Runge-Kutta integration scheme. The system solved is composed of Eqs. (5) and (7) and quaternion derivatives described by Suares (2005), forming a set of 10 equations.

\section{Sphere}

The computation of the sphere trajectory is an interesting case for numerical method verifications due to its simplicity. As the sphere is symmetric, the only significant aerodynamic force is drag, since the rotation is not considered. The global reference axis 
$(X, Y, Z)$ and the local sphere's axis are parallel to the global reference at all times. The definition of the relative velocity, of the angle of attack, and of the side slip angles are the same as in the plate case (see Eqs. (2) and (3)). The drag acts at the center of gravity, and is parallel to the relative velocity. Eq. (13) gives the general expression of the drag:

$$
D=0.5 \rho\left|\mathbf{V}_{\text {rel }}\right|^{2} S C_{d}
$$

where:

- $\quad S$ is the frontal area $\left(\mathrm{m}^{2}\right)$ which is $\pi \mathrm{r}^{2}$, with $\mathrm{r}$ being the sphere radius;

- $C_{d}$ is the drag coefficient.

Clift, Grace and Weber (1978) give correlations to determine the $C_{d}$ coefficient depending on the Reynolds number $(\mathrm{Re})$. These correlations are shown in Table 1, next.

Table 1. Clift et al.'s (1978) sphere drag coefficient correlations

\begin{tabular}{|c|c|}
\hline Reynolds number range & Empirical $C_{d}$ value \\
\hline $\operatorname{Re}<0.01$ & $\frac{3}{16}+\frac{24}{\mathrm{Re}}$ \\
\hline $0.01<\mathrm{Re} \leq 20$ & $24\left(1+0.1315 \mathrm{Re}^{0.82-0.05 \log (\mathrm{Re})}\right)$ \\
\hline $20<\operatorname{Re} \leq 260$ & $\begin{array}{c}\mathrm{Re} \\
\frac{24\left(1+0.1935 \mathrm{Re}^{0.6305}\right)}{\operatorname{Re}}\end{array}$ \\
\hline $260<\operatorname{Re} \leq 1500$ & $10^{1.6435-1.1242 \log (\mathrm{Re})+0.1558 \log (\mathrm{Re})^{2}}$ \\
\hline $1500<\operatorname{Re} \leq 12000$ & $10^{-2.4571+2.5558 \log (\mathrm{Re})-0.9295 \log (\mathrm{Re})^{2}+0.1049 \log (\mathrm{Re})^{3}}$ \\
\hline $12000<\operatorname{Re} \leq 44000$ & $10^{-1.9181+0.6370 \log (\mathrm{Re})-0.0636 \log (\mathrm{Re})^{2}}$ \\
\hline $44000<\operatorname{Re} \leq 338000$ & $10^{-4.3390+1.5809 \log (\mathrm{Re})-0.1546 \log (\mathrm{Re})^{2}}$ \\
\hline $338000<\mathrm{Re} \leq 400000$ & $29.78-5.3 \log (\mathrm{Re})$ \\
\hline $400000<\operatorname{Re} \leq 1 \cdot 10^{6}$ & $0.1 \log (\mathrm{Re})-0.49$ \\
\hline $\operatorname{Re}>1 \cdot 10^{6}$ & $0.19-\frac{8 \cdot 10^{4}}{\operatorname{Re}}$ \\
\hline
\end{tabular}


Finally, the three aerodynamic forces acting on the sphere along each direction are (Eq. (14)):

$$
\begin{gathered}
F_{x}=\operatorname{sign}\left(u_{r}\right) 0.5 \rho\left|\mathbf{V}_{\text {rel }}\right|^{2} S C_{d} \cos (\alpha) \cos (\beta) \\
F_{y}=0.5 \rho\left|\mathbf{V}_{\text {rel }}\right|^{2} S C_{d} \cos (\alpha) \sin (\beta) \\
F_{z}=0.5 \rho\left|\mathbf{V}_{\text {rel }}\right|^{2} S C_{d} \sin (\alpha)
\end{gathered}
$$

Because $\alpha$ and $\beta$ are defined as $\sin ^{-1}$, their values lie between $-90^{\circ}$ and $90^{\circ}$. Thus, $\cos (\alpha)$ and $\cos (\beta)$ are always positive. To avoid having $F_{x}$ always positive, the sign function is used to align the force with the relative velocity direction.

\section{Results and discussion}

In this section, the models described in the previous section will be used to perform trajectory simulations around the BWB. Trajectory patterns around the airplane geometry will be shown, as well as footprints of the trajectories in a transverse plane $20 \mathrm{~m}$ downstream from the nose of the BWB. Prior to these simulations, the code results will be compared and validated against the results of Richards et al (2008) and Tachikawa (1983), for the plate, and against those of Holmes (2004), for the sphere. Both dynamic moment models for the plate will be compared to see the differences in trajectories, and finally, the

difference in footprint distribution behind the BWB between the two models will be highlighted. 
Validation

Plate

- Trajectory code with hybrid dynamic moment (Richards et al 2008)

Only a few articles in the literature present detailed $(X, Y, Z)$ coordinates for computed $3 \mathrm{D}$ trajectories of 6 DOF plates. The single trajectory numerical pattern presented by Richards et al (2008) is used for verification. For this test case, the conditions used are:

- Plate with $S R=2,0.0225 \mathrm{~m}^{2}$ area, i.e., $l=0.1061 \mathrm{~m}$ and $L=0.2122 \mathrm{~m}$, and $e=$ $0.002 \mathrm{~m}$;

- Surface density of $0.340 \mathrm{~kg} / \mathrm{m}^{2}$, corresponding to a density of $170 \mathrm{~kg} / \mathrm{m}^{3}$;

- $10 \mathrm{~m} / \mathrm{s}$ uniform flow field in $X$ direction;

- Initial orientation of the plate: $\Psi=30^{\circ}, \theta=20^{\circ}, \Phi=30^{\circ}$ (see Fig.4).

Comparisons are shown in Fig.8. These two graphs show the trajectory in the $X Z$ and $X Y$ planes, respectively. The trajectory code results are obtained with the model presented in the preceding sections. The differences observed are due to the sensitivity of the trajectory pattern regarding the $C_{N}$ value. Sensitivity tests carried out showed that a $3 \%$ variation in the $C_{N}$ value leads to a difference of about $8 \%$ for the final $Y$ or $Z$ coordinate. The trajectory simulated is longer than the test case proposed by Richards et al, and provides an idea of the trend of the path after $3 \mathrm{~m}$ in $X$, since the reference pattern is short. The dynamic moment used was the one proposed by Richards et al (2008). 


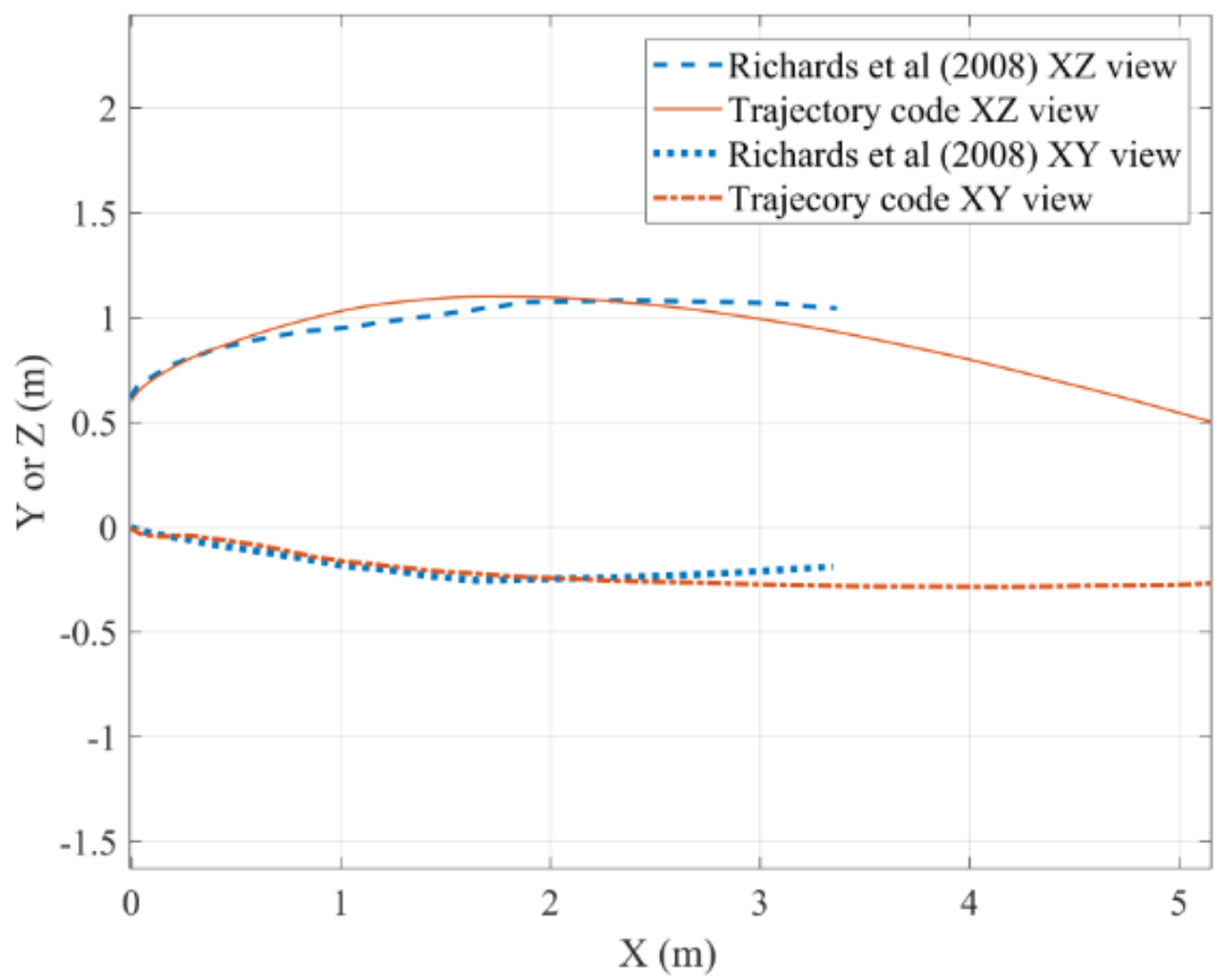

Fig.8 Comparison with the trajectory of Richards et al (2008)

The 3D plate trajectory calculations present satisfactory agreement with the results from the literature: the overall maximum difference observed is $9 \mathrm{~cm}$ in the $X Z$ plane for $X=$ $1.32 \mathrm{~m}$, which represents less than $7 \%$ of variations with respect to the $X$ distance travelled.

○ Tri-linear dependent dynamic moment based on Tachikawa (1983) model

A 3D extension of the 2D dynamic moment was developed based on the model proposed by Tachikawa (1983), in parallel with the implementation of the Richards et al (2008) model. The new model developed in this paper will be called the 3D tri-linear model. Since Tachikawa worked on 2D trajectories, results presented in his paper provide 2D side views 
of trajectories in the $X Z$ plane for different initial pitching angles of the plate. The conditions of the two test cases used to validate the 3D tri-linear model are:

- Square plate with $L=0.04 \mathrm{~m}$ side length, thickness $e=0.002 \mathrm{~m}$, density 1120 $\mathrm{kg} / \mathrm{m}^{3}$

- $X$-unidirectional horizontal wind at $9.2 \mathrm{~m} / \mathrm{s}$;

- Initial pitching angles $\theta_{0}=45^{\circ}$ and $\theta_{0}=75^{\circ}$.

The comparisons are presented in the following figure, where the experimental curves from Tachikawa's video recordings are digitized from his paper, along with the results of his experimental tests.

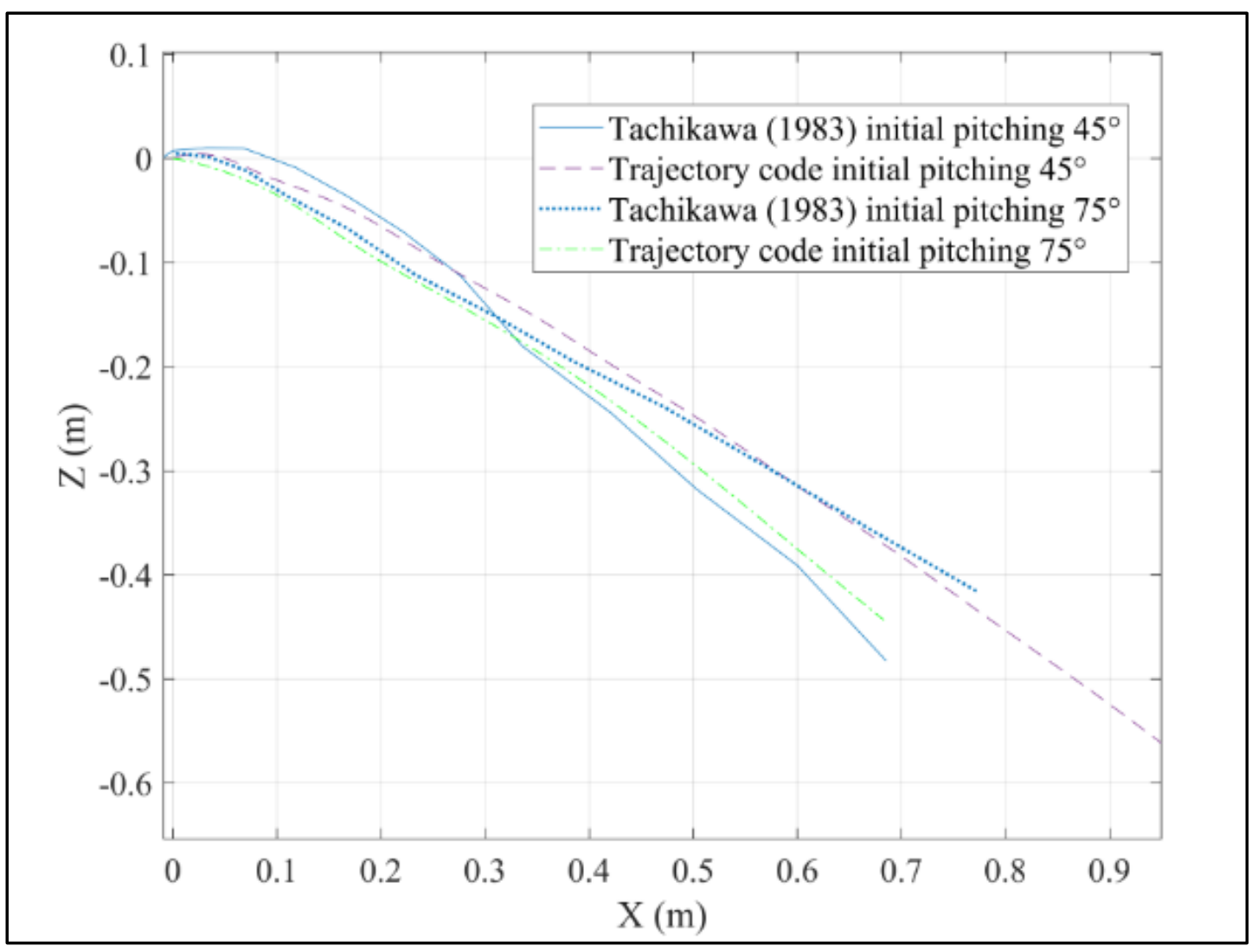

Fig.9 Comparison with Tachikawa's (1983) results, with initial pitching angles of $45^{\circ}$ and $75^{\circ}$ 
The maximum difference observed with respect to the horizontal distance travelled is between 13 and $15 \%$. These differences are acceptable since the trajectory code model uses the correlation of Richards et al (2008) for the aerodynamic normal coefficient. Tachikawa did not use the same model for the aerodynamic coefficient, but rather, adapted it depending on the dynamic effects. In the present 3D tri-linear model, this dependence is neglected. To highlight the capability of the 3D code, the previous test case, with an initial angle of $75^{\circ}$, was reproduced with a $Y$-unidirectional wind. The initial angle of release was about the $X_{p}$ axis:

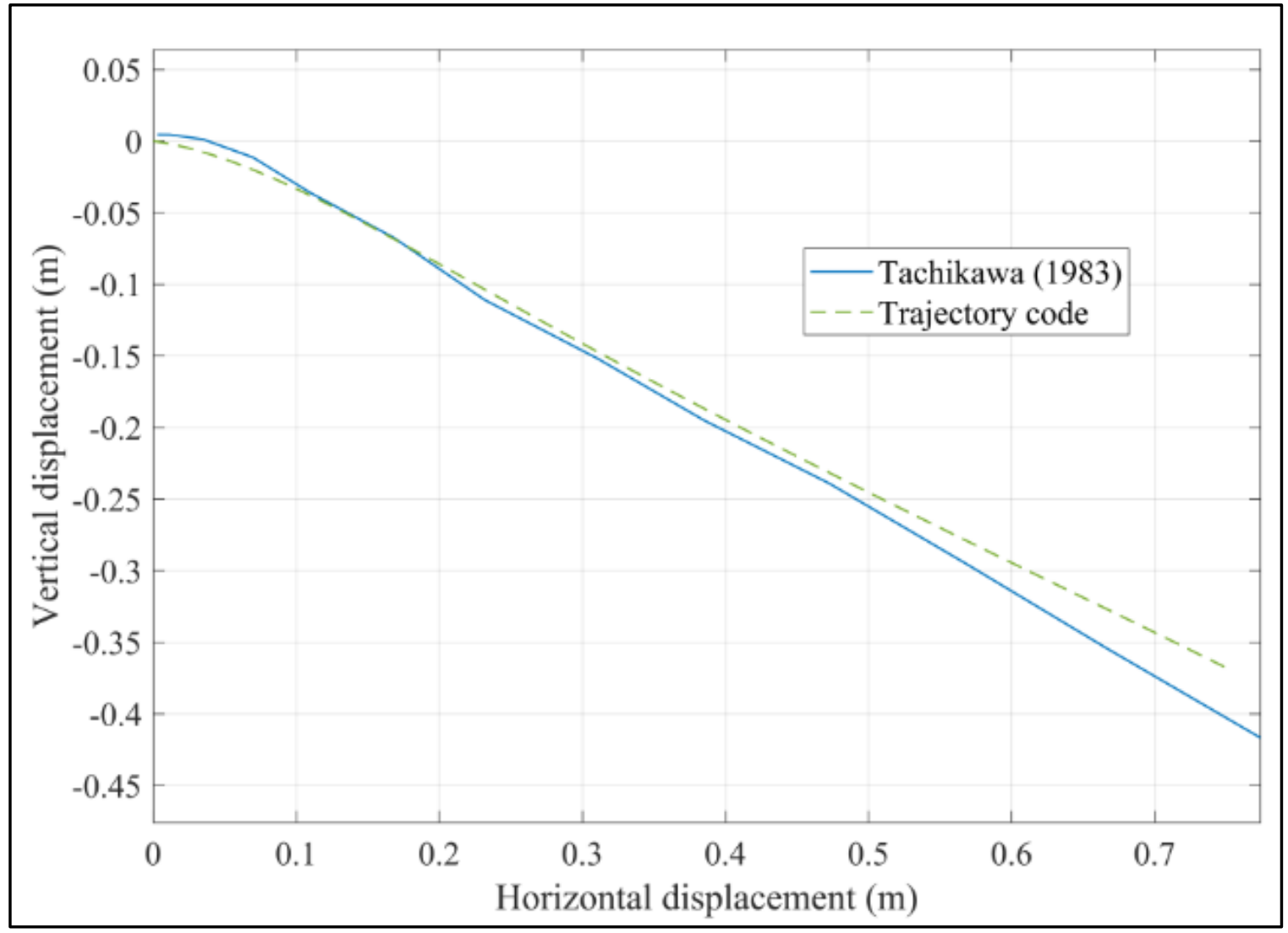

Fig.10 Side view comparison, with a $Y$-unidirectional wind 
With a wind in the $Y$ direction, the pattern is still in accordance with the observations of Tachikawa, presenting less than $5 \%$ in differences.

It is interesting to compare both dynamic moment models for the same test case. The following figures present comparisons for the test case used for the verification of the dynamic Richards et al model. Fig.11 shows comparisons of the trajectories, while Fig.12 presents a comparison of the dynamic moment value and the angular velocity.

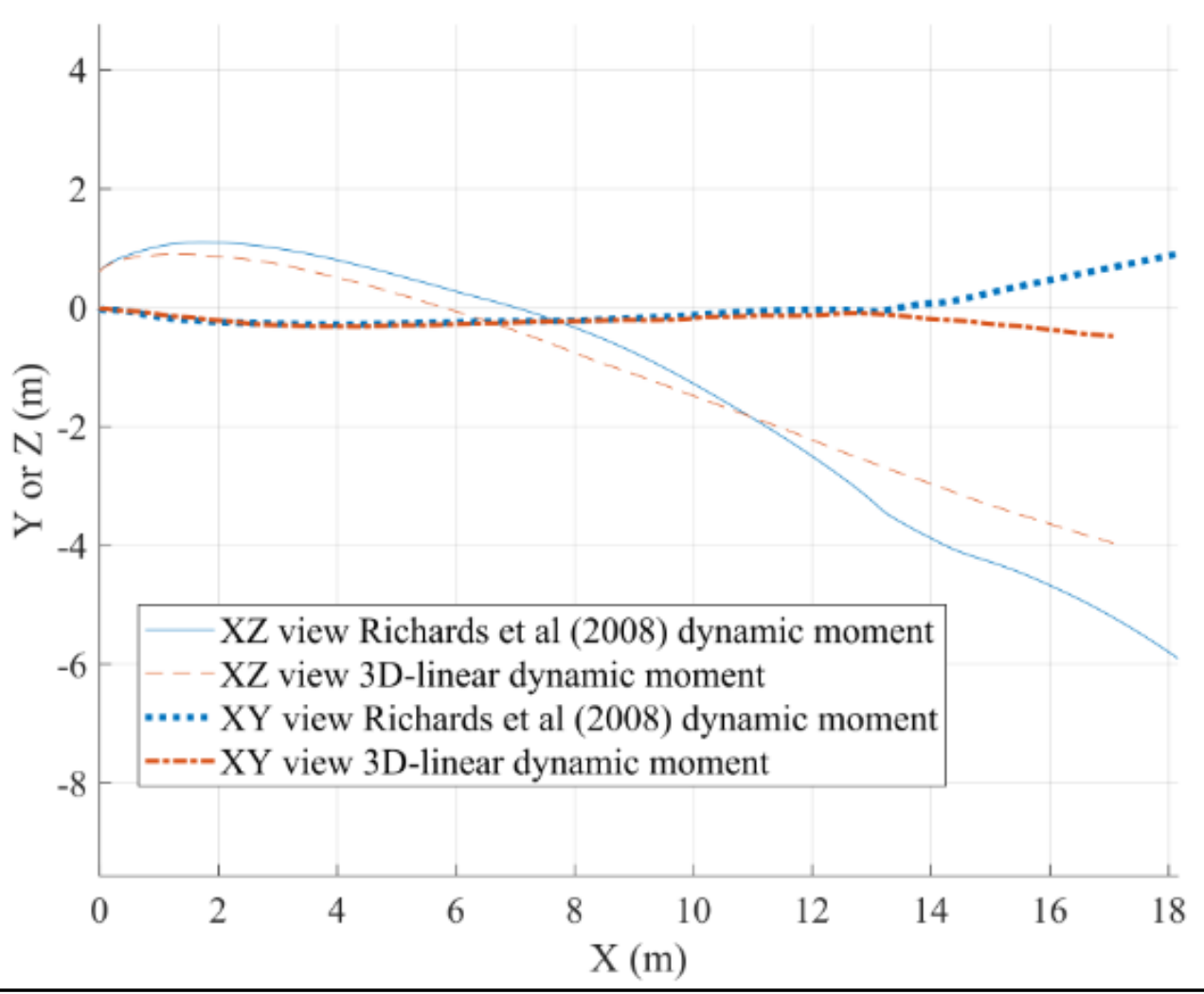

Fig.11 Trajectory comparisons between the two dynamic moment models 


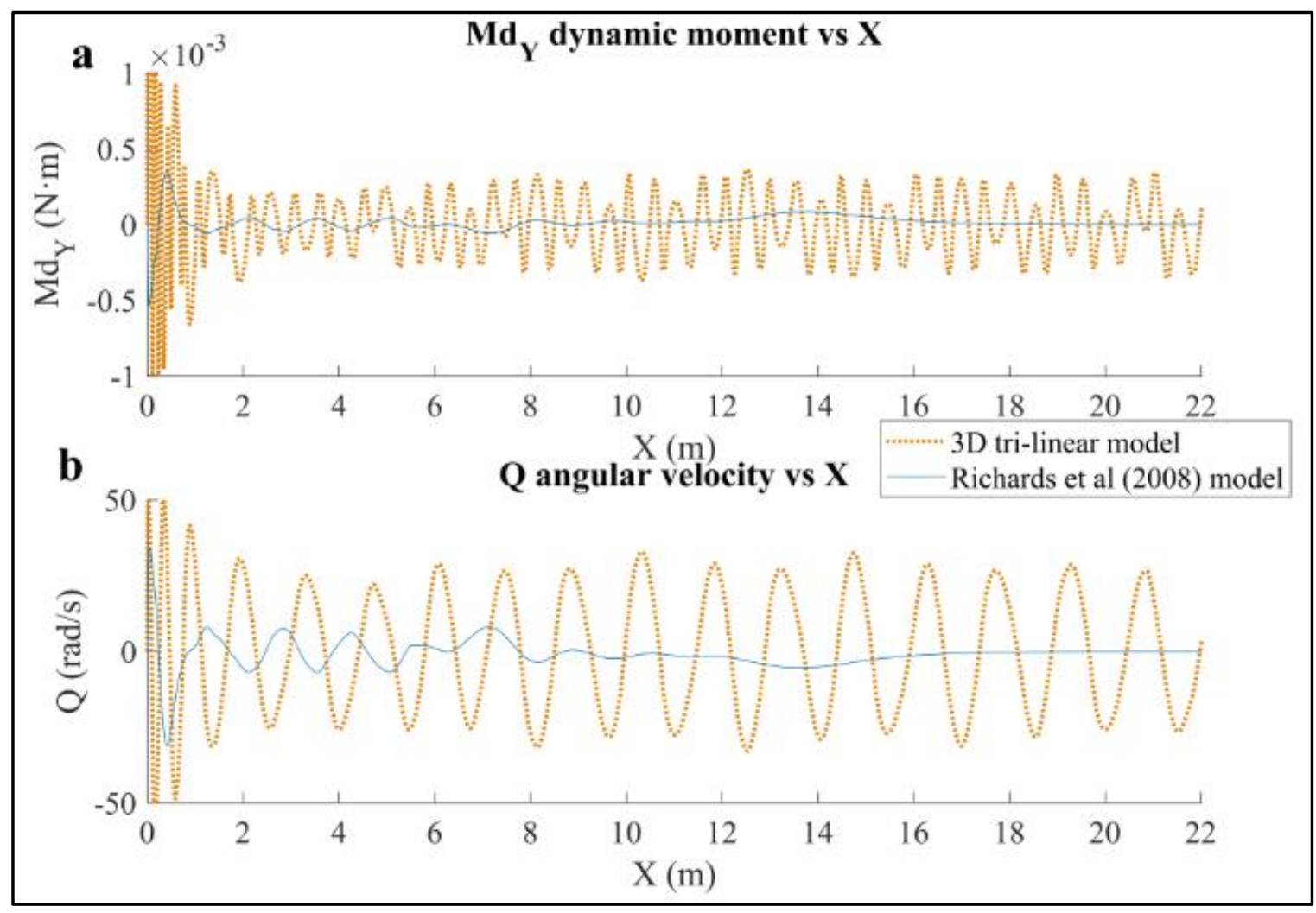

Fig.12 Dynamic moment value (a) and angular velocity (b) comparison

As expected with the definitions of the moments given in the theory section, the moment proposed by Richards et al has a sign constantly opposed to the angular velocity, while the 3D tri-linear dynamic moment model presents a moment with the same sign as the angular velocity. These discrepancies lead to the angular velocity being 0 in the Richards et al case, and blocks the rotation of the plate after a certain period. The same observation was made for the other two rotations about the $X_{p}$ and $Z_{p}$ axes. While the plate is rotating, both models have a similar trajectory. Once the plate stops rotating in the case of Richards et al (around $14 \mathrm{~m}$ in $X$ direction), it obviously leads to an increase in the differences between the trajectories. It would therefore seem that the Richards et al model is suitable for a short simulation distance before the plate stops rotating. Such differences can be explained using the equations describing the models. The Richards et al model, shown in Eq. (6), is mainly 
a damping moment that most often damps the rotation of the plate. The 3D tri-linear model, described in Eqs. (10) and (12), will mostly aid in this rotation, only damping when the limiting angular velocity of Eq. (11) is reached. The 3D tri-linear model is better suited for longer travelled distances, a quality that is required for aeronautics applications.

\section{Sphere}

In the case of the sphere, past studies show 2D results in uniform flow fields. For verification of this paper's 3D code, the uniform flow field is oriented in all three directions to ensure that the results are consistent, prior to comparisons with the literature. The trajectory code results are compared to the results of Holmes (2004) for shed spheres in hurricanes. The test case from Holmes has the following conditions:

- $0.080 \mathrm{~m}$ diameter sphere;

- Wooden sphere of $500 \mathrm{~kg} / \mathrm{m}^{3}$ density;

- Constant unidirectional wind speed of $30.0 \mathrm{~m} / \mathrm{s}$;

- Constant drag coefficient of the sphere at 0.5 , but the correlation of the theory section (Clift et al 1978) is kept in the trajectory code to ensure that the code developed for aeronautics applications is consistent.

The following figures present the comparison with Holmes (2004): Fig.13 plots the horizontal displacement and the horizontal sphere speed versus time. Fig.14 presents the side view of the trajectory seen in the $X Z$ plane. 




Fig.13 Comparison of sphere velocity and displacement with Holmes (2004) results 


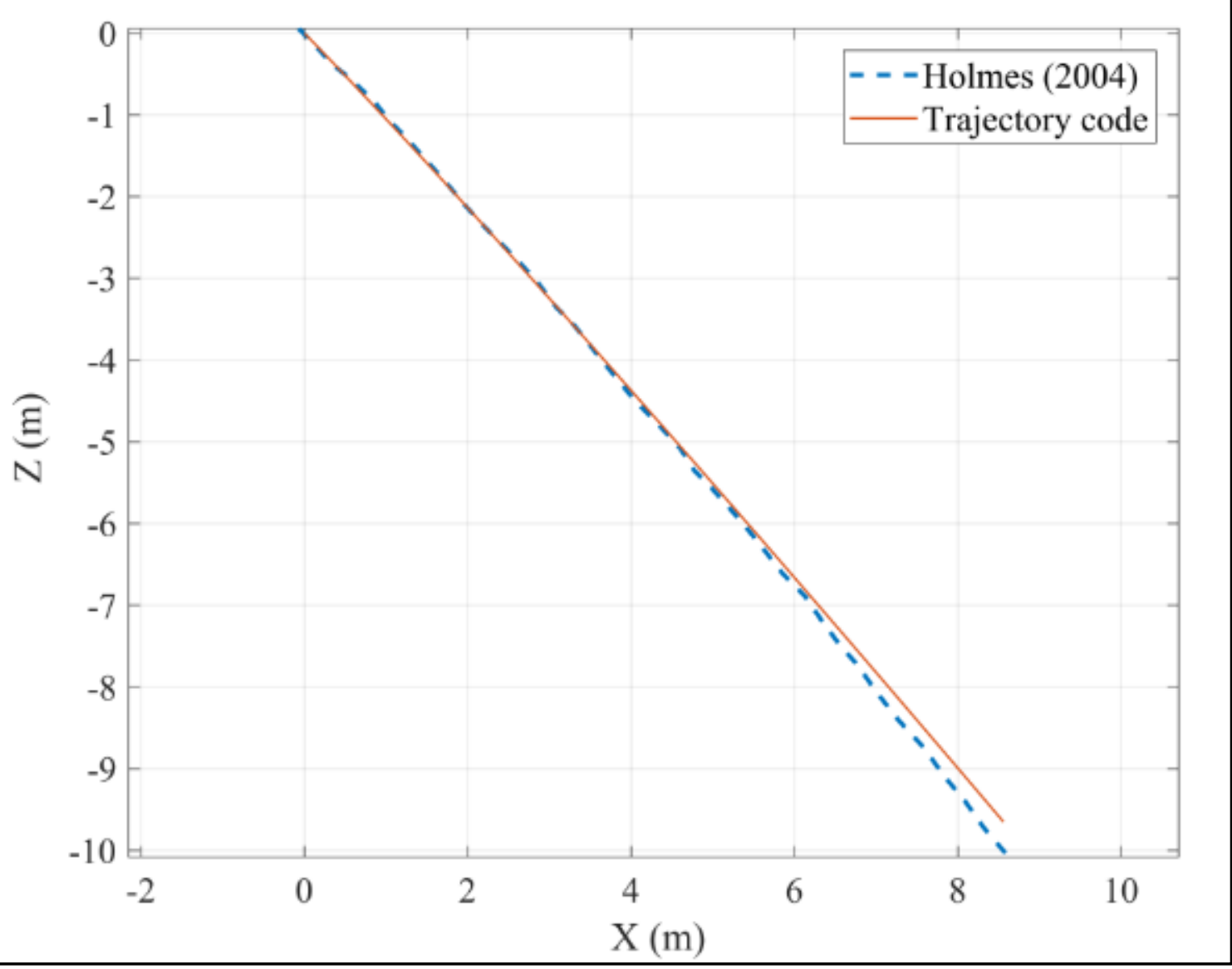

Fig.14 Trajectory comparison in $X Z$ plane with Holmes (2004) results

The results match the curves from the literature perfectly on some portions, and the maximum differences observed at $t=1.4 \mathrm{~s}$ in Fig. 13 and $X=8.5 \mathrm{~m}$ in Fig.14 are acceptable. The 3D sphere trajectory code shows less than $4 \%$ of differences for the biggest gaps, at the end of the trajectory pattern, and is in accordance with the literature, despite not having the same drag coefficient correlation. Holmes chose to keep it constant at 0.5 , while the trajectory code uses the correlation depicted in the theory section (Clift et al 1978). Nevertheless, the correlation used gives a drag coefficient of about 0.5 for the Reynolds numbers reached in the validation test case. At $t=1.6 \mathrm{~s}$, the relative velocity is $9.4 \mathrm{~m} / \mathrm{s}$, which corresponds to a drag coefficient of 0.4674 . This decrease in the drag coefficient 
value along the trajectory can explain the small differences at the end of the graphs of Figs.13 and 14.

Trajectories around the BWB

Plate

Trajectory calculations are done in the flow field described in the methodology section to demonstrate the capability of the code in a non-uniform aerodynamic flow. The plate is a square with a side length of $0.1 \mathrm{~m}$. The results are presented as $(X, Y, Z)$ plots in Fig. 15 . The initial conditions and location of the plate for each of the simulations presented were chosen randomly among 300 trajectories. For clarity, only four trajectories are shown in Fig. 15. Note that the dynamic moment used for these simulations is the 3D tri-linear model:

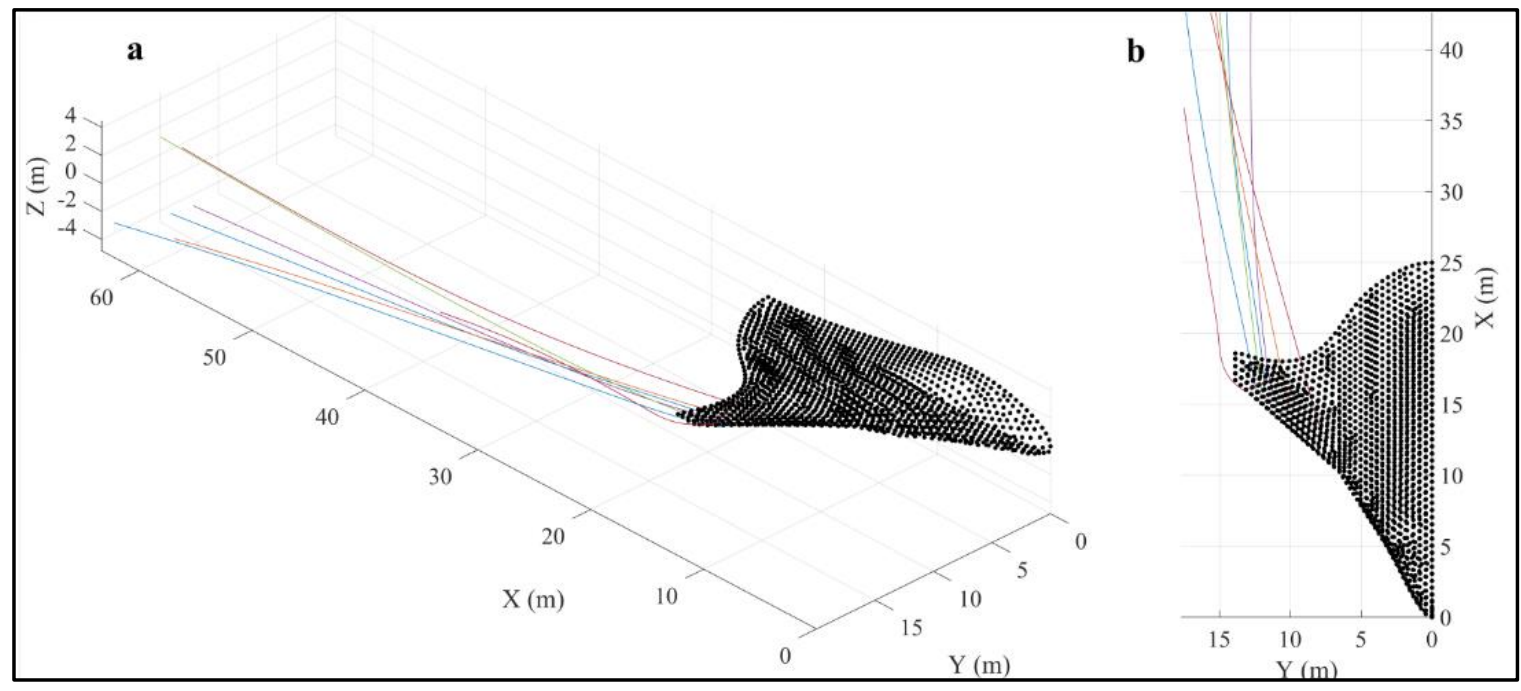

Fig.15 Trajectories of plates around the BWB: 3D view (a) and top view (b)

An analysis of the flow field next to the wing shows a non-negligible $Y$ velocity of the flow, meaning that the aerodynamic forces are responsible for the plates shed from the wing having a visible $Y$ translation component. The gravity has only minor effects on the trajectory. 
Sphere

Other verification test cases were done with a $0.04 \mathrm{~m}$ diameter sphere in the same flow field. Table 2 presents the initial conditions of the trajectory patterns shown on the left side of Fig.16. These initial conditions differ from those in the plate case to avoid collisions with the airplane surface. In the case of collisions, a post-treatment of the trajectories ensures plotting only the part of the trajectories before the impacts instead of going through the surface. Thus, the initial conditions presented in Table 2 are cases that ensure a trajectory without collisions against the BWB.

Table 2. Initial conditions of the sphere simulations

\begin{tabular}{|c|c|c|}
\hline Simulation & $\begin{array}{l}\text { Initial position } X Y Z \\
(\mathrm{~m})\end{array}$ & $\begin{array}{l}\text { Initial velocity } u v w \\
(\mathrm{~m} / \mathrm{s})\end{array}$ \\
\hline Simu 1 & $\begin{array}{lll}15.07 & 12.05 & 0.0600\end{array}$ & $-0.200 \quad 0 \quad 0.200$ \\
\hline Simu 2 & $\begin{array}{lll}16.70 & 13.89 & 0.143\end{array}$ & $\begin{array}{lll}-0.200 & 0 & 0.200\end{array}$ \\
\hline Simu 3 & $\begin{array}{lll}10.71 & 6.93 & 0.105 \\
\end{array}$ & $-0.200 \quad 0 \quad 0.500$ \\
\hline
\end{tabular}

In the case of the sphere, only the drag acts, parallel to the flow velocity. The sphere trajectory should then present a pattern close to, but not totally identical to, the streamlines of the flow field due to inertia and gravity. Fig.16 presents a comparison between the trajectories and the streamlines, as seen from above the BWB. The right view shows the streamlines in the flow field near the initial location of Simulations 1 and 2. 


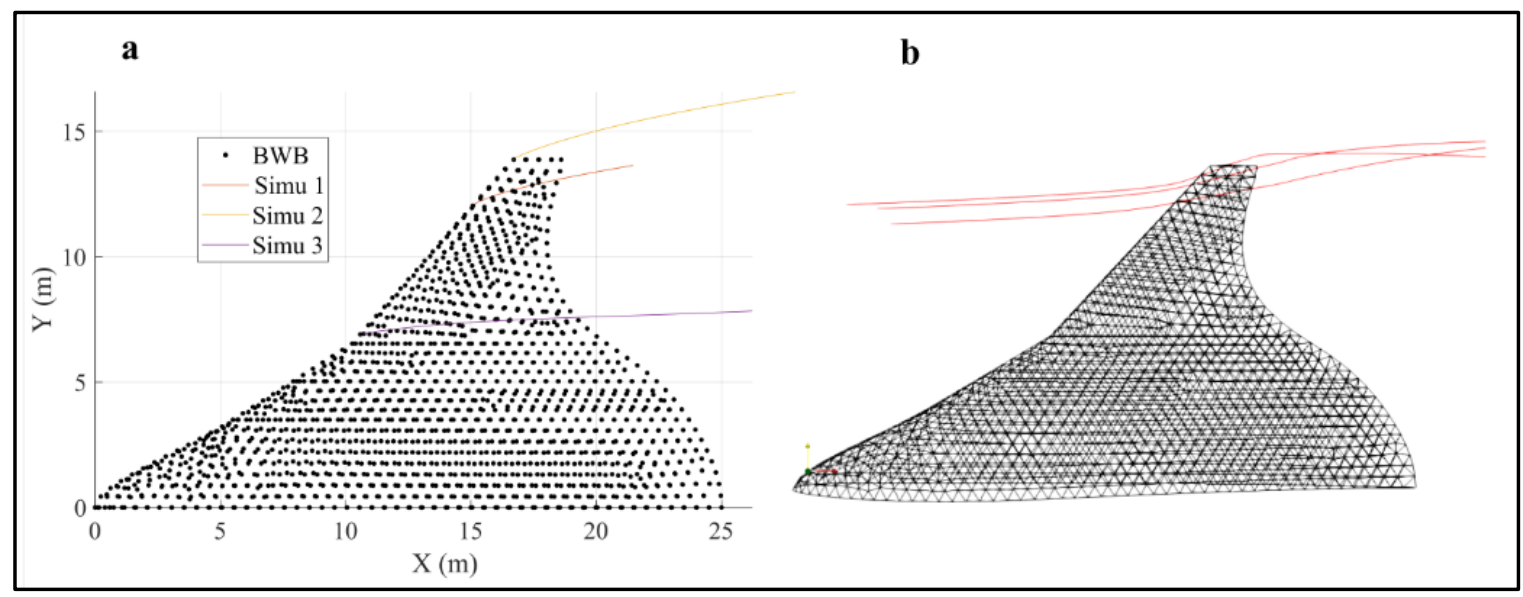

Fig.16 Comparison between the spheres trajectories (a) and the streamlines (b)

Fig.16 shows that the trajectories of Simulations 1 and 2 present a similar orientation as the streamlines passing around the end of the wing. This comparison allows verification of the trajectory code results for the sphere at the tip of the wing. The streamlines move away from the center body once they get near to and downstream from the wing leading edge. The sphere trajectories show the same tendency, although the sphere inertia removes some curvature to the path.

Footprints in a transverse plane

It is interesting to note the effect of the dynamic model choice on the footprints of the trajectories in a cross-plane near the rear of the aircraft. Fig.17 shows footprints of ice blocks in the $Y Z$ cross-plane located $20 \mathrm{~m}$ downstream from the nose of the BWB for both dynamic moment models. It should be noted that the view of the airplane in Fig.17 does not show the section by the cross-plane, but rather, the global rear view of the BWB. A sample of 500 initiated particles was used. All ice particles were released at different locations on the wing since these locations are more likely to present ice accretion in real situations. The range of positions on the wing in the $Y$ direction were between 6.0 and 13.9 
$\mathrm{m}$ and between 9.5 and $16.7 \mathrm{~m}$ in the $X$ direction. The simulations in Fig.17 were carried out with plates with a $0.1 \mathrm{~m}$ side length. All initial conditions were chosen randomly: the position, orientation and initial velocity of the particle were random between 0 and $0.5 \mathrm{~m} / \mathrm{s}$ in both the $X$ and $Z$ directions.

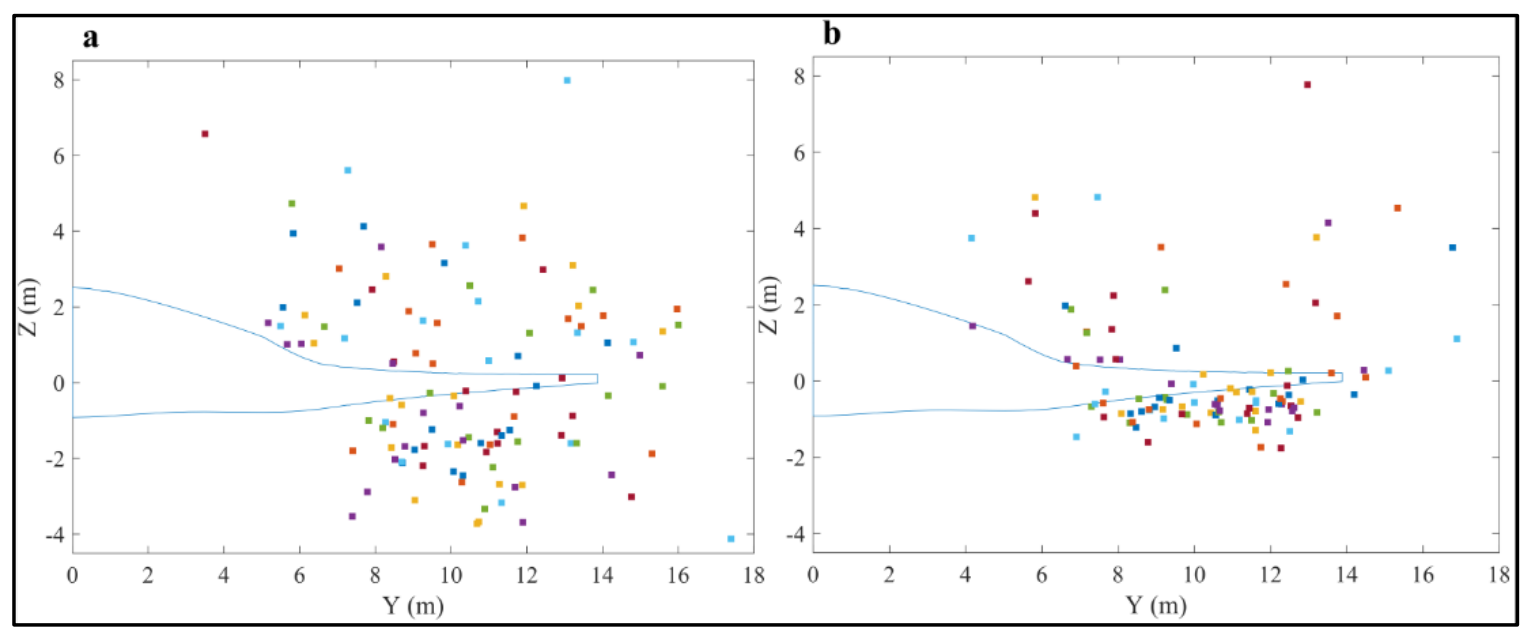

Fig.17 Plates' footprints using the Richards et al (2008) model (a) and the 3D tri-linear model (b)

The following figure converts the previous raw footprints into a probability map, highlighting the zones with a high chance of plate crossings. It can be seen that the mapping differs from one model to the other, confirming the trend seen with the raw footprints. The tri-linear model tends to spread the plate less and gets the high probability zones closer to the aircraft than does the Richards et al model. 


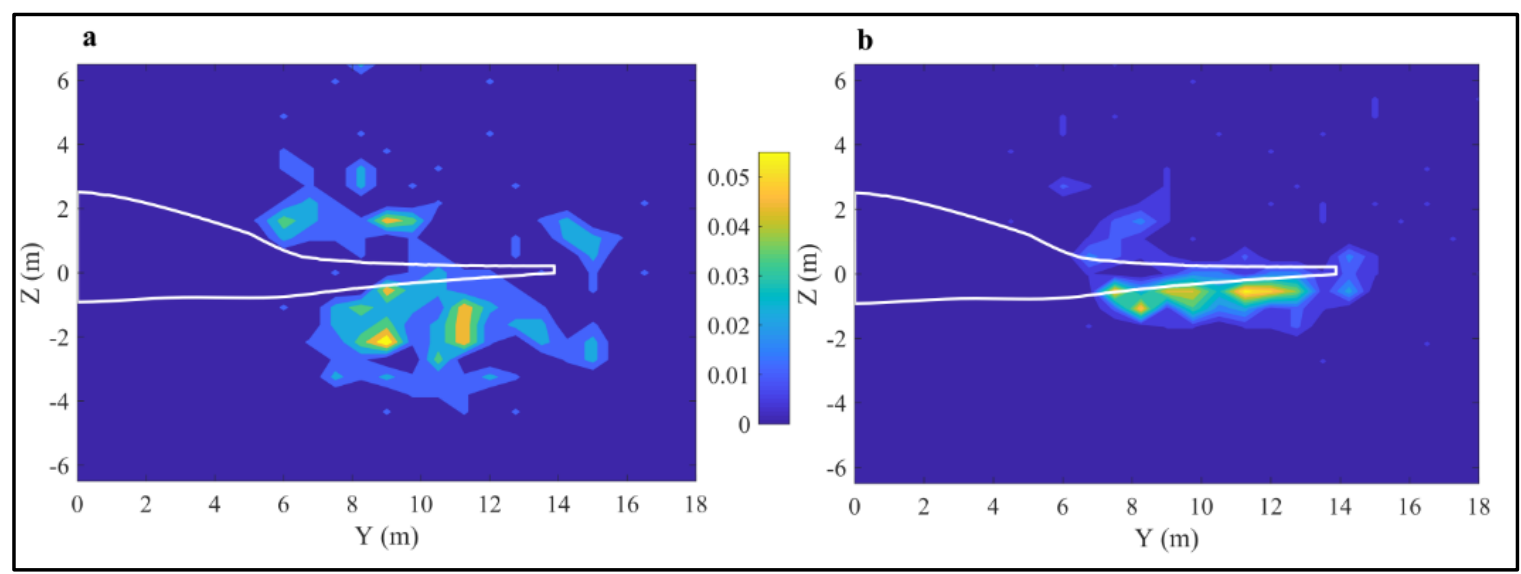

Fig.18 Plates' probability map location using the Richards et al (2008) model (a) and the 3D tri-linear model (b)

Differences are observed between the two dynamic moment models. The Richards et al model, blocking the rotations as seen earlier, presents a wider dispersion, especially in the $Z$ direction. This is explained by the fact that once blocked in rotation in the first case, the plate continues in a privileged direction, depending on the final and fixed orientations. In the case of the proposed 3D model, the plate can rotate freely and adapt its orientation to the flow field. The footprint shows a trajectory concentration just below the wing. Note that about 38 to $44 \%$ of ice blocks reach the $X=20 \mathrm{~m}$ plane, meaning 56 to $62 \%$ of blocks strike the aircraft. The computed trajectories are sensitive to the choice of the dynamic moment model. For real ice fragments, it is to be expected that the constant $K$ and $\omega_{0}$ should be fixed using numerical or experimental databases.

The results shown demonstrate the ability of the code to generate footprints. These observations are not definitive, and further work on probabilistic trajectories, with around $10^{4}$ footprints, has to be carried out to obtain statistically valid conclusions regarding the footprint distribution and validate an engine location.

\section{Conclusions}


During the development phase of an aircraft, anticipating ice ingestion and impacts by numerical trajectory simulation is crucial. A three-dimensional 6 DOF Lagrangian approach models the trajectory of the sphere and plate ice particles. The innovation in the approach in the present work lies in developing a 3D tri-linear dynamic moment model based on previous $2 \mathrm{D}$ work in order to allow the plate to rotate during its complete movement. The solution of the equations of motion and quaternion equations to model the particle motion and rotations implied the use of an interpolation module to compute the flow velocity at each point from a CFD solution. In a Lagrangian frame of reference, correlations from the literature determine the static aerodynamic coefficients. Finally, the code is in good agreement with previous works in wind engineering. As a proof of concept, the code is used to study footprint sensitivity to the dynamic model selection around a blended wing body aircraft in a low speed phase. Future work will focus on dynamic model improvement for the plate and ice fragments using CFD. The statistical study of the trajectories and the related uncertainties will also be used for the verification of highfidelity methods based on immersed boundary methods.

\section{Acknowledgements}

This work was supported by ISAE-ENSMA, Poitiers, France; AéroÉTS, Montreal, Canada and the Office of the Dean of Studies, ÉTS, Montreal, Canada.

\section{References}

Amidror, I. (2002). Scattered data interpolation methods for electronic imaging systems: a survey. Journal of Electronic Imaging., 11(2), 20.

Baker, C. J. (2007). The debris flight equations. Journal of Wind Engineering and Industrial Aerodynamics, 95(5), 329-353. doi:10.1016/j.jweia.2006.08.001 
Beaugendre, H., Nouveau, L., Costes, M., Wervaecke, C., \& Kilian, T. (2018). High-fidelity methods for the prediction of ice debris trajectories 2018 AIAA Aerospace Sciences Meeting: American Institute of Aeronautics and Astronautics.

Clift, R., Grace, J. R., \& Weber, M. E. (1978). Bubbles, Drops, and Particles: Academic Press. Conversy, S., Chatty, S., Gaspard-Boulinc, H., \& Vinot, J.-L. (2014). The Accident of Flight AF447 Rio-Paris: a Case Study for HCl Research. Paper presented at the IHM'14, 26e conférence francophone sur l'Interaction Homme-Machine, Lille, France. https://hal.archivesouvertes.fr/hal-01089633

Fu, A. M., Huang, P., \& Gu, M. (2013). Numerical model of three-dimensional motion of platetype wind-borne debris based on quaternions and its improvement in unsteady flow. Paper presented at the 2nd International Conference on Civil, Architectural and Hydraulic Engineering, ICCAHE 2013, July 27, 2013 - July 28, 2013, Zhuhai, China.

He, Y., Bayly, A. E., \& Hassanpour, A. (2018). Coupling CFD-DEM with dynamic meshing: A new approach for fluid-structure interaction in particle-fluid flows. Powder Technology, 325, 620-631. doi:10.1016/j.powtec.2017.11.045

Hoerner, S. F. (1965). Fluid-dynamic drag; practical information on aerodynamic drag and hydrodynamic resistance. Midland Park, N.J.

Holmes, J. D. (2004). Trajectories of spheres in strong winds with application to wind-borne debris. Journal of Wind Engineering and Industrial Aerodynamics, 92(1), 9-22. doi:10.1016/j.jweia.2003.09.031

I.A.T.A. (2016). Safety Report 2015. Retrieved from Montreal, Quebec:

Ignatowicz, K., \& Morency, F. (2017). Numerical Study of Ice Particle Shedding: Interpolation Methods and 2D Trajectories. Paper presented at the CASI AERO 2017, Toronto, ON, Canada.

https://www.openconf.org/aero2017/modules/request.php?module $=0 \mathrm{C}$ proceedings \& action=summary.php\&id=184\&a=Accept

Kordi, B., \& Kopp, G. A. (2009). Evaluation of Quasi-Steady Theory Applied to Windborne Flat Plates in Uniform Flow. Journal of Engineering Mechanics, 135(7), 657-668. doi:doi:10.1061/(ASCE)EM.1943-7889.0000008

Nichols, J. O. (1977). Analysis and compilation of missile aerodynamic data. Volume I, Data presentation and analysis. Washington, D.C.: National Aeronautics and Space Administration Springfield, Va.

Olivier, M., \& Dumas, G. (2012). Numerical Strategies for Partitioned Fluid-Structure Interaction Algorithms. Paper presented at the 20th Annual Conference of the CFD Society of Canada, Canmore, Canada.

Palacios, F., Economon, T. D., \& Alonso, J. J. (2015). Large-scale aircraft design using SU2 53rd AIAA Aerospace Sciences Meeting: American Institute of Aeronautics and Astronautics.

Papadakis, Hsiung-Wei., Y., \& G., S. I. (2007). Simulation of Ice Shedding from a Business Jet Aircraft. Paper presented at the 45th AIAA Aerospace Sciences Meeting and Exhibit.

Papadakis, M., Yeong, H.-W., \& Shimoi, K. (2007). Parametric Investigation of Ice Shedding from a Business Jet Aircraft. Paper presented at the SAE Aircraft \& Engine Icing International Conference, Seville, Spain.

Paramasivam, S., Sugendran, R., \& Anbarasan, T. (2015). Aerodynamic Configuration Design of a Missile (Vol. V4).

Richards, P. J., Williams, N., Laing, B., McCarty, M., \& Pond, M. (2008). Numerical calculation of the three-dimensional motion of wind-borne debris. Journal of Wind Engineering and Industrial Aerodynamics, 96(10), 2188-2202. doi:10.1016/j.jweia.2008.02.060 
Sefastsson, U. (2016). Evaluation of Missile Guidance and Autopilot through a 6 DOF Simulation Mode. (Master), KTH Royal Institute of Technology, Stockholm.

Shampine, L. F., \& Reichelt, M. W. (1997). The MATLAB ODE Suite. SIAM Journal on Scientific Computing, 18(1), 1-22. doi:10.1137/s1064827594276424

Skeen Jr, J. T., \& Reed, S. L. (2004). Weather-related aviation accident investigations at the national transportation safety board. Paper presented at the 11th Conference on Aviation, Range, and Aerospace Meterology, October 4, 2004 - October 8, 2004, Hyannis, MA, United States.

Suares, I. G. (2005). Ice Particle Trajectory Simulation. (M. Sc. Master thesis), Wichita State University, Wichita.

Tachikawa, M. (1983). Trajectories of flat plates in uniform flow with application to windgenerated missiles. Journal of Wind Engineering and Industrial Aerodynamics, 14(1), 443-453. doi:https://doi.org/10.1016/0167-6105(83)90045-4

Védie, L., Morency, F., \& Kubler, N. (2016). Sensitivity analysis of ice piece trajectory calculation 8th AIAA Atmospheric and Space Environments Conference: American Institute of Aeronautics and Astronautics.

Velázquez Salazar, O., Weiss, J., \& Morency, F. (2017). Preliminary investigation on stall characteristics of a Regional BWB for low speed approach. Paper presented the 35th AIAA Applied Aerodynamics Conference. 